\title{
Hard and bright gamma-ray emission at the base of the Fermi bubbles
}

\author{
L. Herold and D. Malyshev
}

Erlangen Centre for Astroparticle Physics, Erwin-Rommel-Str. 1, Erlangen, Germany
e-mail: laura.herold@fau.de; dmitry.malyshev@fau.de

Received 16 November 2018 / Accepted 1 April 2019

\begin{abstract}
Context. The Fermi bubbles (FBs) are large gamma-ray emitting lobes extending up to $55^{\circ}$ in latitude above and below the Galactic center (GC). Although the FBs were discovered eight years ago, their origin and the nature of the gamma-ray emission are still unresolved. Understanding the properties of the FBs near the Galactic plane may provide a clue to their origin. Previous analyses of the gamma-ray emission at the base of the FBs, what remains after subtraction of Galactic foregrounds, have shown an increased intensity compared to the FBs at high latitudes, a hard power-law spectrum without evidence of a cutoff up to approximately $1 \mathrm{TeV}$, and a displacement of the emission to negative longitudes relative to the GC.

Aims. We analyze nine years of Fermi Large Area Telescope data in order to study in more detail than the previous analyses the gamma-ray emission at the base of the FBs, especially at energies above $10 \mathrm{GeV}$.

Methods. We used a template analysis method to model the observed gamma-ray data and calculate the residual emission after subtraction of the expected foreground and background emission components. Since there are large uncertainties in the determination of the Galactic gamma-ray emission toward the GC, we used several methods to derive Galactic gamma-ray diffuse emission and the contribution from point sources to estimate the uncertainties in the emission at the base of the FBs.

Results. We confirm that the gamma-ray emission at the base of the FBs is well described by a simple power law up to $1 \mathrm{TeV}$ energies. The $95 \%$ confidence lower limit on the cutoff energy is about $500 \mathrm{GeV}$. It has larger intensity than the FBs emission at high latitudes and is shifted to the west (negative longitudes) from the GC. If the emission at the base of the FBs is indeed connected to the highlatitude FBs, then the shift of the emission to negative longitudes disfavors models in which the FBs are created by the supermassive black hole at the GC. We find that the gamma-ray spectrum can be explained either by gamma rays produced in hadronic interactions or by leptonic inverse Compton scattering. In the hadronic scenario, the emission at the base of the FBs can be explained either by several hundred supernova remnants (SNRs) near the GC or by about ten SNRs at a distance of $\sim 1 \mathrm{kpc}$. In the leptonic scenario, the necessary number of SNRs that can produce the required density of CR electrons is a factor of a few larger than in the hadronic scenario.
\end{abstract}

Key words. astroparticle physics - Galaxy: center - gamma rays: diffuse background - Galaxy: bulge - ISM: jets and outflows ISM: bubbles

\section{Introduction}

The Fermi bubbles (FBs) are one of the most spectacular and unexpected discoveries in the Fermi Large Area Telescope (LAT) data (Su et al. 2010). The FBs extend up to $55^{\circ}$ in latitude above and below the Galactic center (GC), they have a well-defined edge and a relatively uniform intensity across the surface, apart from a "cocoon" in the southeastern part of the bubbles (Su \& Finkbeiner 2012; Ackermann et al. 2014). The intensity spectrum is $\sim E^{-2}$ at gigaelectronvolt energies with a cutoff or a softening around $100 \mathrm{GeV}$ at latitudes $|b|>10^{\circ}$ (Ackermann et al. 2014). The origin of the FBs is attributed either to an emission from the supermassive black hole (SMBH) at the center of our Galaxy or to a period of starburst activity that resulted in a combined wind from supernova (SN) explosions of massive stars ( $\mathrm{Su}$ et al. 2010). The gamma-ray signal up to $100 \mathrm{GeV}$ can be produced either by interactions of hadronic cosmic rays (CRs) with gas (hadronic model) or by inverse Compton (IC) scattering of high-energy electrons and positrons and interstellar radiation (leptonic model).

Although the FBs were detected about eight years ago, their origin is still unresolved. Important insights into their origin can be obtained from the study of the morphology and the spectrum of the FBs near the GC. The spectrum of gamma rays can provide information on the composition of the CRs that produce the gamma-ray signal (hadronic versus leptonic CRs), as well as the age of the CRs (through a cooling cutoff in the leptonic scenario or a break from the escape of high-energy CRs) and the spectrum of the CRs at the source. The morphology of the emission can point to the source of the bubbles: either the SMBH Sgr A* or a recent star-forming region. Previous analyses of the FBs at low latitudes indicated higher intensity of emission near the Galactic plane (GP) and a displacement to negative longitudes (Acero et al. 2016; Ackermann et al. 2017; Storm et al. 2017). The spectrum of the FBs for $|b|<10^{\circ}$ is consistent with a power-law $\propto E^{-2}$ without a cutoff up to $1 \mathrm{TeV}$ (Ackermann et al. 2017).

The study of the FBs in the GP is complicated owing to bright Galactic diffuse emission components. The $\pi^{0}$ component of the gamma-ray emission is well traced by the distribution of gas. But this component has large uncertainties toward the GC because of a lack of kinematic information from the motion of the gas in the Galaxy, which is used to reconstruct the gas distribution; the velocity of the gas in the direction of the GC is perpendicular to the line of sight. These uncertainties are also a consequence of the uncertainties in $\mathrm{CO}$ emission along the line of sight to the GC, which is used as a tracer of molecular 
hydrogen, and large dispersion of velocities of some molecular clouds near the GC. There are also large uncertainties in the distribution of the CR sources and the propagation model near the GC, which make it rather difficult to predict a priori the distribution of the propagated CRs in the Galaxy. For the IC component of the gamma-ray emission, there is a significant uncertainty in the interstellar radiation field (ISRF) density near the GC (Popescu et al. 2017; Porter et al. 2017; Niederwanger et al. 2019) in addition to the uncertainties in the CR distribution. There should also be undetected point-like and extended sources, which nevertheless contribute to the total flux. The distribution of CRs in the Galaxy can be computed with CR propagation tools, such as GALPROP (Strong et al. 2007). The maps of gamma-ray emission from interactions of CRs with gas in different Galactocentric rings and from interaction of CR electrons and positrons with the ISRF can be used as templates for the corresponding components of gamma-ray emission. The agreement of the gamma-ray data with models based on templates derived with the CR propagation tools is rather poor in the GP (e.g., Porter et al. 2017).

In this paper, we analyze the gamma-ray emission at the base of the FBs and estimate the uncertainties related to modeling of the Galactic foreground and background components. We focus on the morphology and spectrum of the FBs at energies from $10 \mathrm{GeV}$ to $1 \mathrm{TeV}$, for which the intensity of the gamma-ray emission from the FBs relative to the other Galactic components is higher than at low energies as a consequence of softer spectra of the Galactic components. The study of the FBs at high energies will be also important for future searches with neutrino and Cherenkov telescopes, such as the Cubic Kilometer Neutrino Telescope (KM3NeT) and the Cherenkov Telescope Array (CTA).

We use several methods to determine the Galactic foreground and background emission. Unfortunately the notion of the foreground and background emission may not be well defined if we search for an extended gamma-ray emitting source based on the gamma-ray data itself rather than using multiwavelength data, which are used, for example, to trace the distribution of gas. In this work, by foreground and background emission we mean a steady state (or average) diffuse emission of gamma-rays. The steady state distribution of CRs is obtained by averaging in time over many sources; it results in the local CR proton spectrum of $\sim E^{-2.7}$ for energies from $\sim 5 \mathrm{GeV}$ to $\sim 1 \mathrm{PeV}$ and $\sim E^{-3.3}$ for the local CR electrons from $\sim 1 \mathrm{GeV}$ to $\sim 1 \mathrm{TeV}$ energies. If the spectrum of the CRs at the source is $\sim E^{-2.0-2.2}$, then the softening of the spectrum of CR protons by $\sim E^{-0.3}-E^{-0.6}$ is due to energy-dependent escape from the Galaxy, while the softening for the CR electrons by $\sim E^{-1}$ is due to cooling. A distinguishing property of a source of CRs is that the spectrum of CRs at or near the source is much harder than the average (propagated) spectrum of CRs. Thus, it is possible to look for the presence of a population of freshly accelerated CRs by searching for areas of gamma-ray emission with a spectrum harder than average. In particular, one can use an "on-off" analysis to subtract the steady state component of the gamma-ray emission. One of the caveats of this analysis is that even the steady state gamma-ray emission in the "on" region can be more intense than the emission in the "off" region, but in this case the difference of the fluxes would have a soft spectrum characteristic of the propagated CRs, unless the difference in flux is much smaller than the flux in both on and off regions. In this case the difference can have a harder spectrum than the two terms. We use the on-off technique as a preliminary check in a search for a population of freshly accelerated CRs: the hard spectrum of the difference is a necessary condition for the presence of a population of CRs with a spectrum harder than the steady state distribution of CRs. It is also a sufficient condition for the presence of a population of CRs with a hard spectrum if the difference has intensity comparable to the overall intensity in the on and off regions.

Consequently, considering that there is a tentative asymmetry in diffuse gamma-ray emission near the GC at high energies with a spectrum harder to the west of the GC relative to the emission to the east of the GC, as a first step, we estimate the amount of the asymmetry by taking the difference in the gamma-ray data to the west and east of the GC after masking bright point sources (PS). Second, since the spectrum of the FBs at high latitudes and the inferred spectrum at low latitudes are harder than the spectra of the other components of diffuse emission, the contribution of the FBs at energies $\lesssim 1 \mathrm{GeV}$ is relatively small. We use the data below $1 \mathrm{GeV}$ as a template of the Galactic foreground emission, which we fit to the data at energies above $1 \mathrm{GeV}$. The residual emission is used to estimate the contribution from the components with spectra harder than the typical spectra of the steady state diffuse gamma-ray components. Finally, we determine a model for the Galactic gamma-ray diffuse emission using templates for the emission components based on GALPROP calculation ${ }^{1}$ (Moskalenko \& Strong 1998; Strong et al. 2000, 2004, 2007; Ptuskin et al. 2006; Porter et al. 2008; Vladimirov et al. 2011). In this model, we allow many free parameters, such as rescaling of the $\pi^{0}$ and bremsstrahlung emission in Galactocentric rings and refitting of bright PS near the GC. With the many free parameters, this model absorbs as much of the gamma-ray emission at the base of the FBs as it can. As a result, the residual emission at the base of the FBs in this model is smaller than the residuals in the other models considered in the paper.

The similarity of the energy spectrum below $100 \mathrm{GeV}$ of the hard and bright emission at the base of the FBs and the FBs at high latitudes, as well as the spatial coincidence of the emission with a continuation of the FBs from high latitudes, make the physical correspondence of the two objects very plausible. Nevertheless, their alignment along the line of sight can be accidental and the distance to the two objects can be different, i.e., the FBs may be above and below the GC while the hard component may be at a closer distance, for example $1 \mathrm{kpc}$, from us so that the two objects would be physically unrelated. Thus, we refer to the hard and bright component as emission at the base of the FBs to keep both possibilities open: that the emission is the base of the FBs and that the emission is at a different location along the line of sight toward the base of the FBs. We discuss possible origins of the hard component of emission in Sect. 5. Section 6 contains conclusions.

\section{Data selection}

We used nine years of the Fermi-LAT Pass 8 Source class events between August 4, 2008 and August 3, 2017 (Fermi Mission Elapsed Time $239557418 \mathrm{~s}-523411376 \mathrm{~s}$ ) with energies between $316 \mathrm{MeV}=10^{2.5} \mathrm{MeV}$ and $1 \mathrm{TeV}$ separated in 21 logarithmic energy bins (six bins per decade). The selection of the events was performed with the standard quality cuts (DATA_QUAL $>0) \& \&($ LAT_CONFIG==1). In order to avoid contamination from gamma rays produced in interactions of CRs in the Earth atmosphere, we selected events with zenith angles $\theta<100^{\circ}$, which is sufficient for energies above $316 \mathrm{MeV}$. We calculated the exposure and point-spread function (PSF) using the Fermi-LAT Science Tools package version

\footnotetext{
http://galprop.stanford.edu
} 

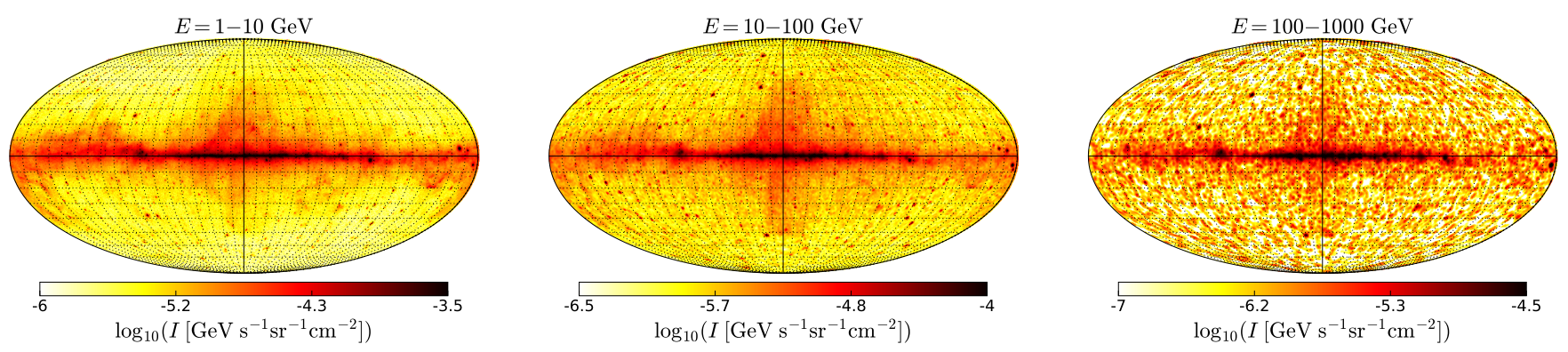

Fig. 1. Energy flux of the Fermi-LAT 9 yr of Source class data integrated in energy ranges $1-10 \mathrm{GeV}, 10-100 \mathrm{GeV}$, and $100 \mathrm{GeV}-1 \mathrm{TeV}$. The maps are represented in logarithmic scale. The map in the energy range $10-100 \mathrm{GeV}(100-1000 \mathrm{GeV})$ is smoothed with a Gaussian kernel with radius $\sigma=0.5(\sigma=0.7)$. The plots are created with the HEALPix package (Górski et al. 2005) in Mollweide projection with nside $=128\left(\approx 0^{\circ} .46\right.$ pixel size).

10-01-01 available from the Fermi Science Support Center ${ }^{2}$ with the P8R2_SOURCE_V6 instrument response functions. Figure 1 shows the energy flux of the gamma-ray emission integrated in three energy ranges ${ }^{3}$. For all maps shown in this paper, we used Galactic coordinates centered on the GC in Mollweide projection. For spatial binning we used the HEALPix ${ }^{4}$ (Górski et al. $2005)$ scheme with a pixelization of order $7(\approx 0.46$ pixel size $)$.

\section{Modeling of the Fermi bubbles at low latitudes}

\subsection{East-west asymmetry in the data}

In order to investigate the asymmetry of the emission at the base of the FBs relative to the GC, we compared the Fermi-LAT data east and west of the GC. We masked the 200 point sources (PS) in the Third Fermi-LAT source catalog (3FGL; Acero et al. 2015) with the largest flux above $1 \mathrm{GeV}$. Each PS is masked with a circle of radius $\frac{\delta}{\sqrt{2}}+0.5$, where $\delta=0.46$ is the characteristic size of the pixels so that $\delta / \sqrt{2}$ is half of the pixel diagonal (if it were a square), while 0.5 is comparable to the $68 \%$ containment radius of the PSF above $\approx 2 \mathrm{GeV}$. Thus the total radius is about 0.82 . In order to avoid possible bias by masking more pixels on one side of the GC in the GP, we symmetrized the PS mask relative to the GC, i.e., we set $m_{-b,-\ell}=0$ if $m_{b, \ell}=0$ and vice versa. We also use this PS mask in the following sections (apart from Sect. 3.4). The data are averaged over regions to the east $\left(0^{\circ}<\ell<10^{\circ}\right)$ and to the west $\left(-10^{\circ}<\ell<0^{\circ}\right)$ of the GC at different latitudes. The regions have a latitude width of $4^{\circ}$. The fraction of masked pixels is about $50 \%$ within $|b|<2^{\circ}$, about $10 \%$ for $2^{\circ}<|b|<6^{\circ}$, and less than $5 \%$ for $|b|>6^{\circ}$.

The difference of the averaged intensity of emission west minus east of the GC is shown in Fig. 2. The error bars represent the statistical errors. The emission for latitudes $b \in\left(-6^{\circ},-2^{\circ}\right)$ and $b \in\left(-2^{\circ}, 2^{\circ}\right)$ shows excess emission to the west of the $\mathrm{GC}$, which remains significant at high energies. The Fermi-LAT exposure within $|b|<10^{\circ}$ and $|\ell|<10^{\circ}$ is rather uniform; the maximal fractional variation of the exposure in this region for $E>1 \mathrm{GeV}$ is less than $4 \%$.

\subsection{Low-energy data as a background model}

In the previous section we have shown that the west-minuseast difference in the Fermi-LAT data in the GP relative to the

\footnotetext{
2 http://fermi.gsfc.nasa.gov/ssc/data/analysis/

3 The integral of the energy flux intensity between $E_{0}$ and $E_{1}$ is defined as $I=\int_{E_{0}}^{E_{1}} E \frac{\mathrm{d} N}{\mathrm{~d} E} \mathrm{~d} E$.

4 http://sourceforge.net/projects/healpix/
}

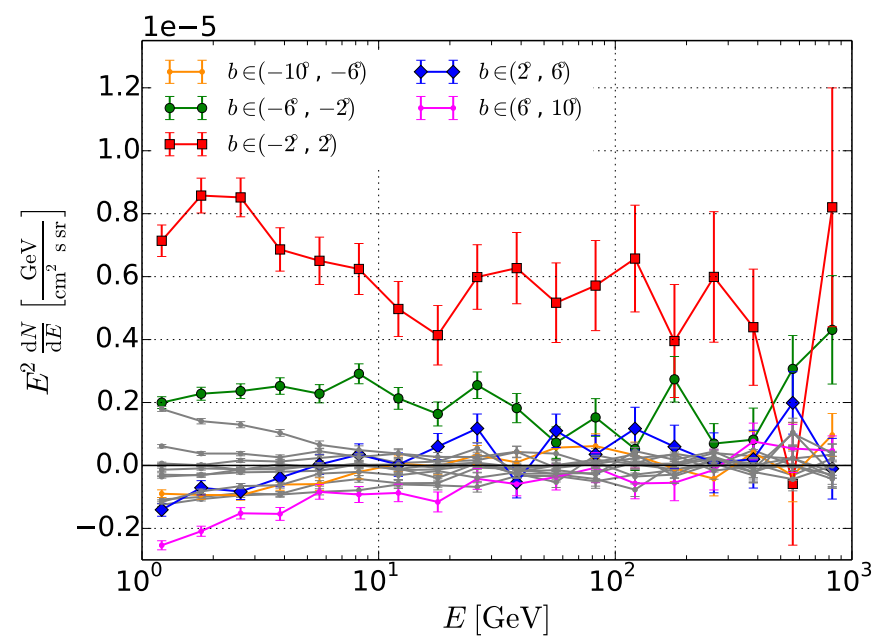

Fig. 2. Difference west minus east in the Fermi-LAT intensity relative to the GC after masking bright PSs. The PS mask is symmetrized relative to the GC. The west (east) region is defined between $-10^{\circ}<\ell<0^{\circ}$ $\left(0^{\circ}<\ell<10^{\circ}\right)$. Gray lines show spectra for latitudes $|b|>10^{\circ}$.

GC has a hard spectrum $\sim E^{-2}$ up to $1 \mathrm{TeV}$. One of the simplest ways to separate the soft component of emission from the hard component of emission is to use the low-energy data as a model of the soft component and subtract those data from the data at higher energies. Gamma rays produced in interactions of the steady state Galactic population of CRs with gas and ISRF, i.e., the $\pi^{0}$, bremsstrahlung, and IC components, dominate the gamma-ray emission in the GP in the energy range $E \lesssim 1 \mathrm{GeV}$. Consequently, the low-energy Fermi-LAT data are a good tracer for the soft components of the diffuse gamma-ray emission in the GP and can be used to create a spatial template for the Galactic foreground. The $68 \%$ containment for the Fermi-LAT photons between $316 \mathrm{MeV}$ and $1 \mathrm{GeV}$ (averaged with an $E^{-2}$ spectrum) is about 1.5 , which is much larger than the sub-degree angular resolution above $1 \mathrm{GeV}$. In order to compensate for the difference in the angular resolution, we smoothed the data in each high-energy bin above $1 \mathrm{GeV}$ with a Gaussian kernel of $\sigma=1^{\circ}$, which corresponds to $68 \%$ containment angle of 1.5 in two dimensions ${ }^{5}$.

We separate the whole sky in latitude stripes of $4^{\circ}$ width where the HEALPix pixels are assigned to stripes that contain

\footnotetext{
5 In general, the width of the Gaussian kernel $\sigma$ can be obtained by subtracting in quadrature the equivalent widths at low and at high energies $\sigma^{2}=\sigma_{\text {low }}^{2}-\sigma_{\text {high }}^{2}$. Since $\sigma_{\text {low }}^{2} \gg \sigma_{\text {high }}^{2}$, we neglect $\sigma_{\text {high }}^{2}$ and smooth the data at high energies with $\sigma_{\text {low }}=1^{\circ}$.
} 

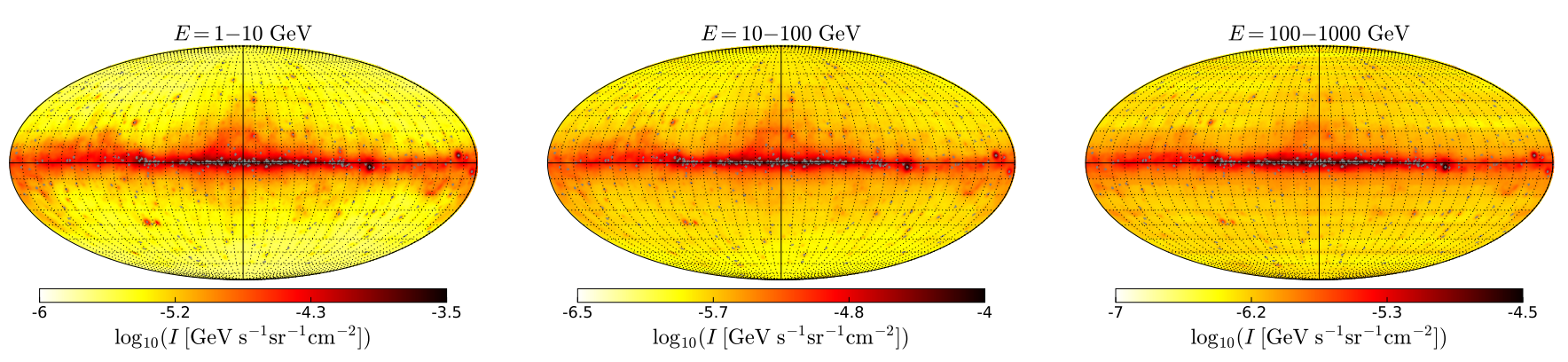

Fig. 3. Energy flux of the diffuse emission model based on the low-energy data (Sect. 3.2) integrated over three energy ranges.
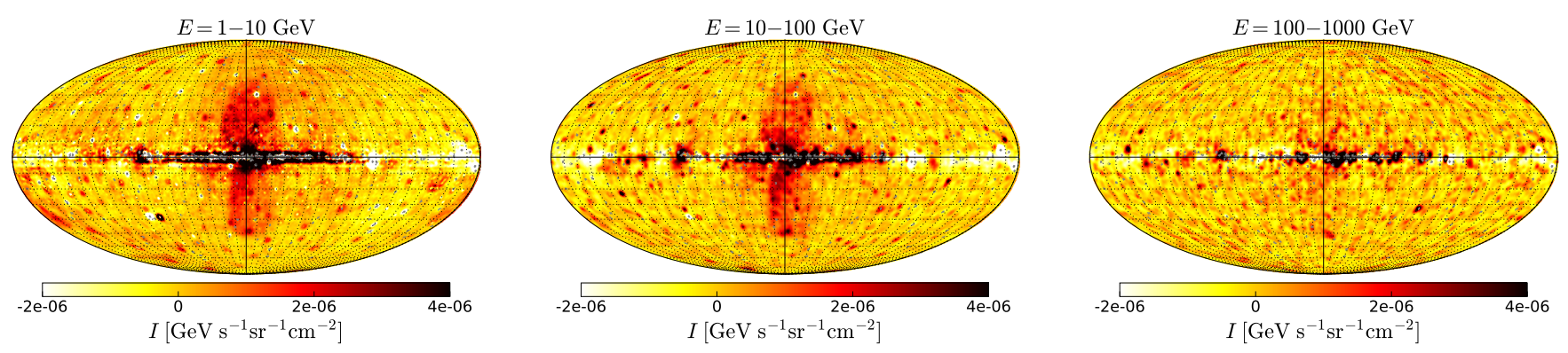

Fig. 4. Energy flux of the residuals of the low-energy model derived in Sect. 3.2 integrated over three energy ranges.

the centers of the pixels. We parametrized the model in each stripe and each energy bin independently, i.e., in the latitude stripe $b$ for energy bin $E$ and HEALPix pixel with index $i$ the model consists of the following two terms:

$M_{b}(E, i)=k_{b}(E) \cdot \tilde{N}_{b}^{\text {low }}(E, i)+c_{b}(E) \cdot \tau(E, i)$,

where $k_{b}(E)$ and $c_{b}(E)$ are coefficients to be determined from the fit to the data. The term $c_{b}(E) \cdot \tau(E, i)$ is proportional to the exposure $\tau(E, i)$ : it accounts for the isotropic extragalactic background and partially compensates for the latitude-dependent IC emission. The term $k_{b}(E) \cdot \tilde{N}_{b}^{\text {low }}(E, i)$ is proportional to the low-energy photon counts summed over $n_{\text {low }}=3$ energy bins between $316 \mathrm{MeV}$ and $1 \mathrm{GeV}$ rescaled by the ratio of exposures at low and at high energies, i.e.,

$\tilde{N}_{b}^{\text {low }}(E, i)=\frac{1}{n_{\text {low }}}\left(\sum_{\alpha=1}^{n_{\text {low }}} \frac{N\left(E_{\alpha}, i\right)}{\tau\left(E_{\alpha}, i\right)}\right) \tau(E, i)$,

where $N\left(E_{\alpha}, i\right)$ is the number of photons in energy bin $E_{\alpha}$ and in pixel $i$.

We determine the parameters $c_{b}(E)$ and $k_{b}(E)$ by fitting the model to the Fermi-LAT data in energy bins $E>1 \mathrm{GeV}$. We maximize the log likelihood of the gamma distribution with respect to parameters $c_{b}(E)$ and $k_{b}(E)$ using the Python iminuit optimizer $^{6}$. In order to avoid an overcompensation for the flux from the FBs, the region $-20^{\circ}<\ell<20^{\circ}$ is excluded from the fit, i.e., the fit is performed for the total remaining length of the stripe $20^{\circ}<\ell<340^{\circ}$. In the following, the region $-20^{\circ}<$ $\ell<20^{\circ}$ is referred to as the region of interest (ROI) of the FBs. Bright PSs are masked with the mask described in Sect. 3.1.

$6 \quad$ The gamma distribution $p(x ; \tilde{k}+1)=\frac{x^{\tilde{k}}}{\Gamma(\tilde{k}+1)} e^{-x}$ is the probability density function for the parameter $x$ given the observed counts $\tilde{k}$. The counts $\tilde{k}$ are integer for unsmoothed maps and non-integer for the smoothed maps. We discuss the optimization of the model parameters using smoothed maps in Appendix B.
After we fit the model in each latitude stripe, we interpolated it inside the ROI of the bubbles. The intensity of the model energy flux integrated in three energy ranges is shown in Fig. 3, while the residuals after subtracting the model from the data are presented in Fig. 4. The FBs are clearly visible in the first two energy ranges, $E=1-10 \mathrm{GeV}$ and $E=10-100 \mathrm{GeV}$. For $E=100 \mathrm{GeV}-1 \mathrm{TeV}$ the statistics are low, but we can still see an excess near the GP. There are other regions of large residuals in the GP and at high latitudes. These are diffuse and point-like sources with spectra harder than the average spectra of Galactic and extragalactic sources. In particular, there is a residual in diffuse emission along the GP within $|\ell| \lesssim 60^{\circ}$ at energies below $100 \mathrm{GeV}$. This residual can be due to IC emission, which has a harder spectrum than the average spectrum of the $\pi^{0}$ component; to a harder spectrum of CRs in the inner Galaxy (Gaggero et al. 2015; Acero et al. 2016; Yang et al. 2016); or to a population of hard PSs in the GP in the inner parts of the Galaxy. At energies above $100 \mathrm{GeV}$ the residuals are resolved into localized sources, some of which are extended. Apart from the Galactic foreground and background components, there are gamma rays emitted by the interactions of CRs with the Sun and its radiation field (Abdo et al. 2011b) and the Moon (Ackermann et al. 2016). The intensities of the emission from the Sun and the Moon are about an order of magnitude less than the intensity of the emission from the FBs. As a result, these components are not visible on the residual maps at energies above $10 \mathrm{GeV}$.

\subsection{Rectangles model of the bubbles}

In the previous subsection, we excluded the ROI of the FBs. In this subsection, we perform the fit over the whole sky and model the emission from the FBs using rectangles. In order to explore the east-west asymmetry of the FBs, we introduce two rectangular templates in each latitude stripe $b$ and energy bin $E$ : one east, $\ell \in\left(0^{\circ}, 20^{\circ}\right)$, and one west, $\ell \in\left(-20^{\circ}, 0^{\circ}\right)$, of the GC. The width of the rectangles is $4^{\circ}$, i.e., the same as the width of the latitude stripes. We use the same foreground model as in Sect. 3.2 plus the isotropic template. Overall, the model has four terms in each 

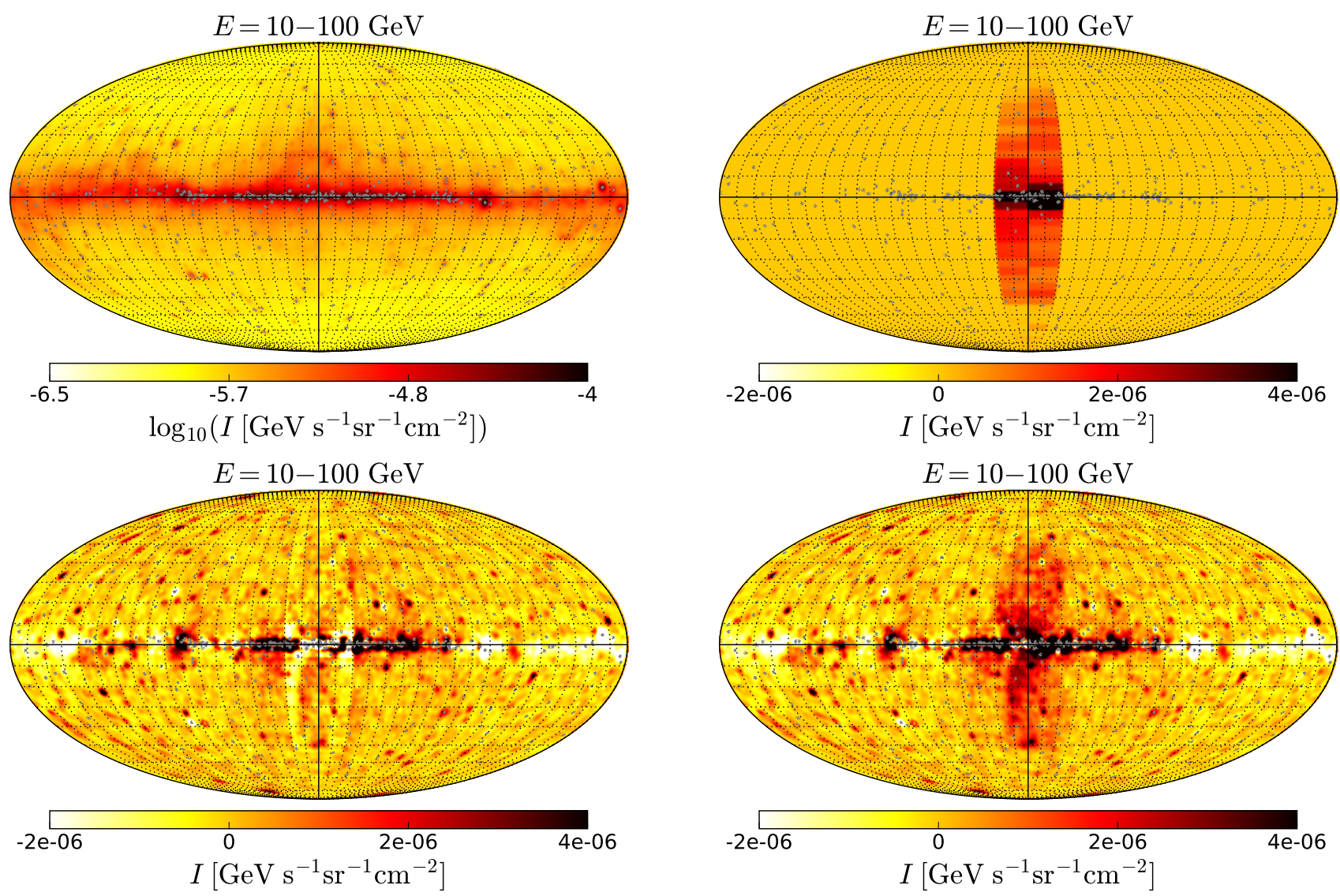

Fig. 5. Energy flux of the diffuse model excluding the rectangles model of the FBs (top left), the rectangles model of the FBs (top right), the residual after subtracting the total model (bottom left), and residuals plus the rectangles model of the FBs (bottom right) derived in Sect. 3.3 integrated between 10 and $100 \mathrm{GeV}$.

energy bin as follows:

$$
\begin{aligned}
M_{b}(E, i)= & k_{b}(E) \cdot \tilde{N}_{b}^{\text {low }}(E, i)+c_{b}(E) \cdot \tau(E, i) \\
& +R_{b}^{\text {east }}(E) S_{b}^{\text {east }}(i)+R_{b}^{\text {west }}(E) S_{b}^{\text {west }}(i),
\end{aligned}
$$

where the scaling parameters $k_{b}(E)$ and $c_{b}(E)$ and the FBs model parameters $R_{b}^{\text {east }}(E)$ and $R_{b}^{\text {west }}(E)$ are determined independently in each $4^{\circ}$ latitude stripe and in each energy bin $E>1 \mathrm{GeV}$ by fitting the model to the smoothed Fermi-LAT data (see Sect. 3.2 for details on the smoothing of the data). The values $S_{b}^{\text {east }}(i)$ and $S_{b}^{\text {west }}(i)$ are step function templates, which are equal to 1 in each latitude stripe $b$ for $0^{\circ}<\ell<20^{\circ}$ and $0^{\circ}>\ell>-20^{\circ}$, respectively, and 0 otherwise. The energy fluxes of the model and the residuals integrated in the range $E=10-100 \mathrm{GeV}$ are shown in Fig. 5.

\subsection{GALPROP model of the foreground and PS refitting}

In this section, we fit the gamma-ray data using a model derived with the GALPROP Galactic CR propagation code v54.1. The model for the diffuse emission components is the same as the "sample" model in Ackermann et al. (2017). In particular, we used the GALPROP code to calculate templates for the Galactic diffuse emission components. We determine five templates in each energy bin for gamma rays produced in interactions of hadronic CRs with gas and bremsstrahlung emission corresponding to five Galactocentric rings: $0-1.5,1.5-3.5,3.5-8,8-10$, and $10-50 \mathrm{kpc}$. We assume propagation halo height of $10 \mathrm{kpc}$ and radius of $20 \mathrm{kpc}$ and spin temperature $150 \mathrm{~K}$. We use three IC templates corresponding to the three ISRF components: cosmic microwave background (CMB), infrared (IR) emission of dust, and starlight (SL). In addition to GALPROP templates, we used a flat template for the FBs at latitudes $|b|>10^{\circ}$ (Ackermann et al. 2014). We model the Loop I feature using a geometric template (e.g., Fig. 2 of Ackermann et al. 2014) based on a polarization survey at $1.4 \mathrm{GHz}$ (Wolleben 2007). Templates for the gammaray emission from the Sun and the Moon (Orlando \& Strong 2008; Abdo et al. 2011b; Johannesson et al. 2013; Ackermann et al. 2016) were obtained with the Fermi Science Tools ${ }^{7}$. We also include, as a GC excess template, dark matter annihilation with a generalized Navarro-Frenk-White (gNFW) profile with index $\gamma=1.25$ (Goodenough \& Hooper 2009; Abazajian et al. 2014; Calore et al. 2015) and scaling radius $r_{\mathrm{s}}=20 \mathrm{kpc}$, as well as the isotropic template to model the non-resolved extragalactic sources.

For the PS, we added sources from the 3FGL catalog (Acero et al. 2015) to form a template in each energy bin. We created independent templates for the Large Magellanic Cloud and the Cygnus region. We also added the remaining extended sources in a separate template. The spatial templates for the extended sources are provided by the 3FGL catalog. The PS mask in this section is different from the mask that we used in the previous sections. In this case, we mask the 200 sources with largest flux above $1 \mathrm{GeV}$ outside a $10^{\circ}$ circle from the GC. Each source is masked with a circle of $\frac{\delta}{\sqrt{2}}+1^{\circ}$ radius $(\delta=0.46$ is the

\footnotetext{
http://fermi.gsfc.nasa.gov/ssc/data/analysis/ scitools/solar_template.html
} 

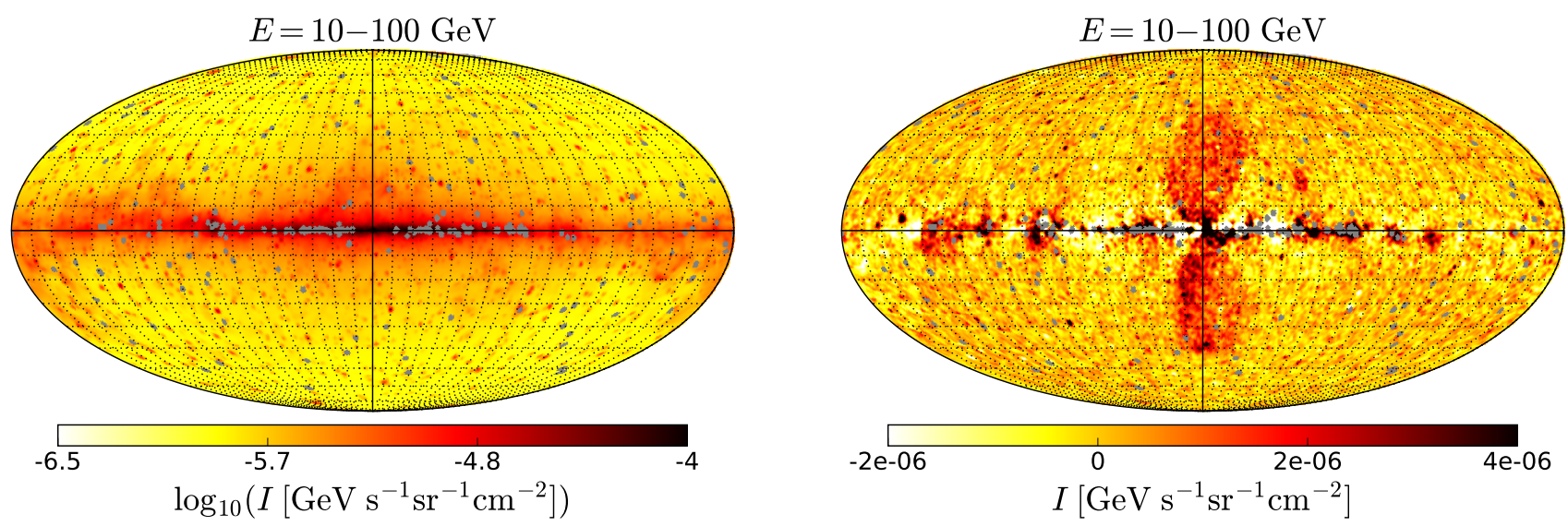

Fig. 6. Energy flux of the background model based on rescaled GALPROP templates (left) and of the residuals plus the FBs and the gNFW component (right) derived in Sect. 3.4 integrated between 10 and $100 \mathrm{GeV}$.
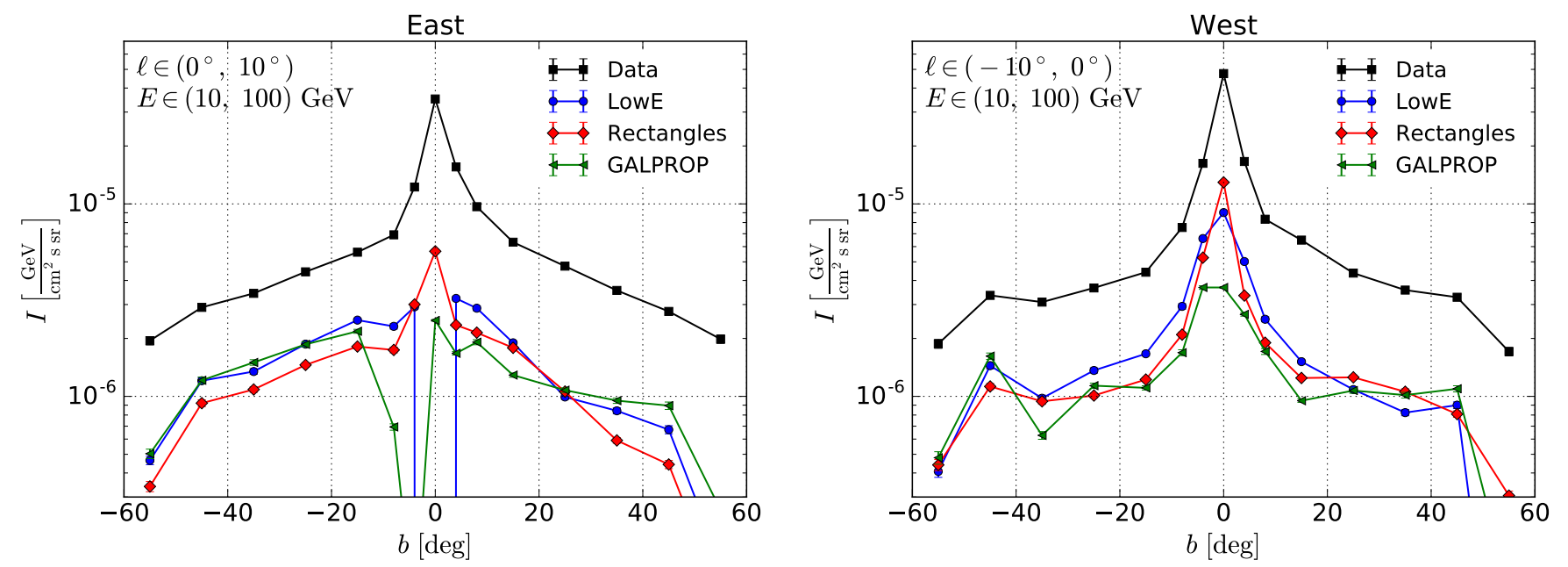

Fig. 7. Latitude profiles of the energy flux between 10 and $100 \mathrm{GeV}$ for the total data excluding the PS mask and for the FBs models for different foreground diffuse emission models to the east (left panel) and to the west (right panel) of the GC.

characteristic size of the pixels), which is larger than the radius of $\frac{\delta}{\sqrt{2}}+0.5$ used in the previous sections. We do not mask PS within $10^{\circ}$ from the GC. Instead, we include independent templates for 40 sources with largest flux between 10 and $100 \mathrm{GeV}$ taken from the column "Flux10000_100000" in the 3FGL cata$\log$. Each source is fit independently to each energy bin, i.e., we do not use the spectral information from the 3FGL catalog. In Fig. 6 we show the residual emission plus the FBs model and the gNFW model summed over energies between 10 and $100 \mathrm{GeV}$. Owing to the model having more free parameters in the GP relative to the models considered in the previous sections, there are fewer positive residuals in the GP. There are, however, negative residuals in several locations, which indicates errors in the model. In particular, there is a rather significant negative residual to the east of the GC. Nonetheless, the FBs are clearly visible along with the bright emission at the base of the FBs, which also displays a shift to negative longitudes relative to the GC.

\section{Morphology and spectrum of the gamma-ray emission at the base of the FBs}

\subsection{Latitude and longitude profiles}

In order to quantitatively estimate the difference in the intensity of the emission of the FBs at high and at low latitudes, as well as the asymmetry of the gamma-ray emission at the base of the FBs, we plot the latitude profiles to the east and west of the GC. In Fig. 7 we show the profiles of the energy flux between $10-100 \mathrm{GeV}$ as functions of the Galactic latitude for different models of Galactic foreground emission. For the low-energy model, the profiles show the residual gamma-ray emission; for the rectangles model the profiles show the intensity of the rectangles templates model. In case of the GALPROP model, we show the sum of the residual emission, the FBs template, and the GC excess template, as described in Sect. 3. For comparison we show the latitude profile of the total gamma-ray data (excluding pixels in the PS mask). For positive longitudes, some models overpredict the gamma-ray data near the GP, which leads to negative residuals. For negative longitudes, there is an increase of flux at the base of the FBs by at least a factor of 2 to 6 for all models relative to the gamma-ray emission from the FBs at high latitudes. The results for the GALPROP model differ from the low-energy and rectangles model in the GP by a factor of 2 to 3 . This is due to additional freedom in the GALPROP model related to the usage of several templates in the GP.

In Fig. 8 we show the longitude profiles of the residual flux for the background model based on low-energy data. The excess at $\ell \approx-13^{\circ}$ for $|b|<2^{\circ}$ is probably due to residual emission around the SNR RX J1713 (Abdo et al. 2011a). Consequently, we do not consider this source as a part of the emission at the 

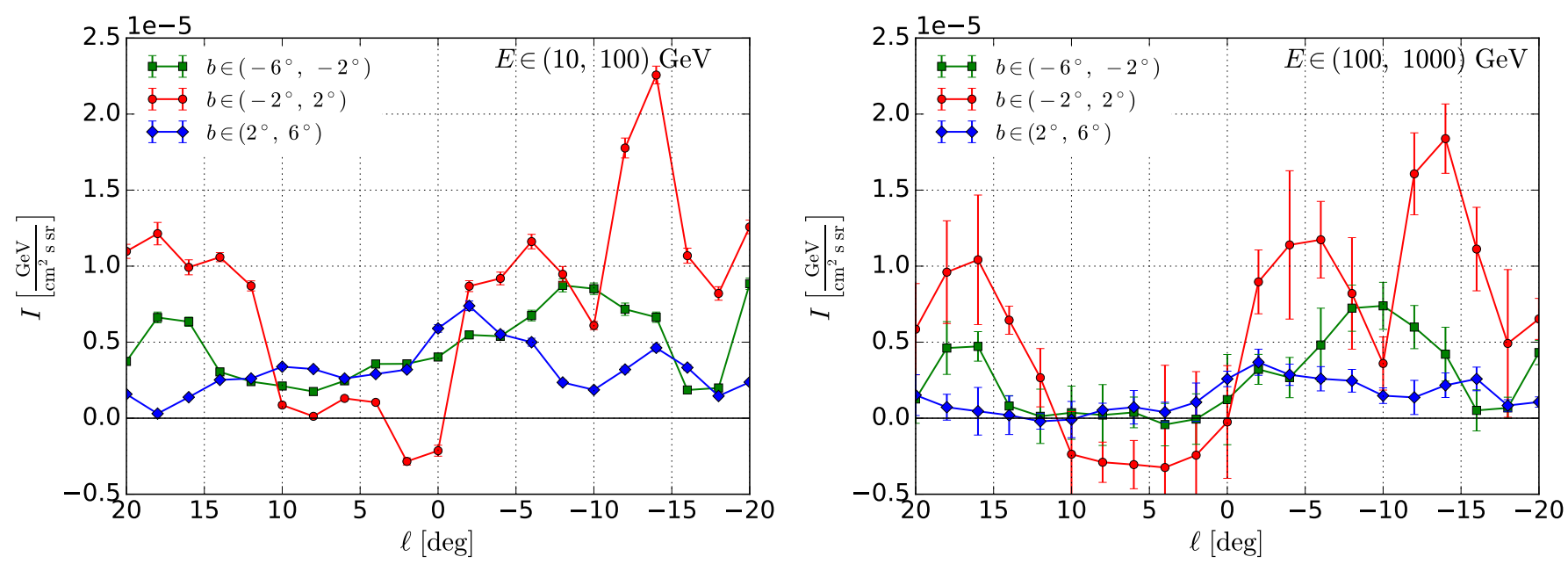

Fig. 8. Longitude profiles of the energy flux between 10 and $100 \mathrm{GeV}$ and between $100 \mathrm{GeV}$ and $1 \mathrm{TeV}$ for the residuals in the low-energy data background model (Sect. 3.2).
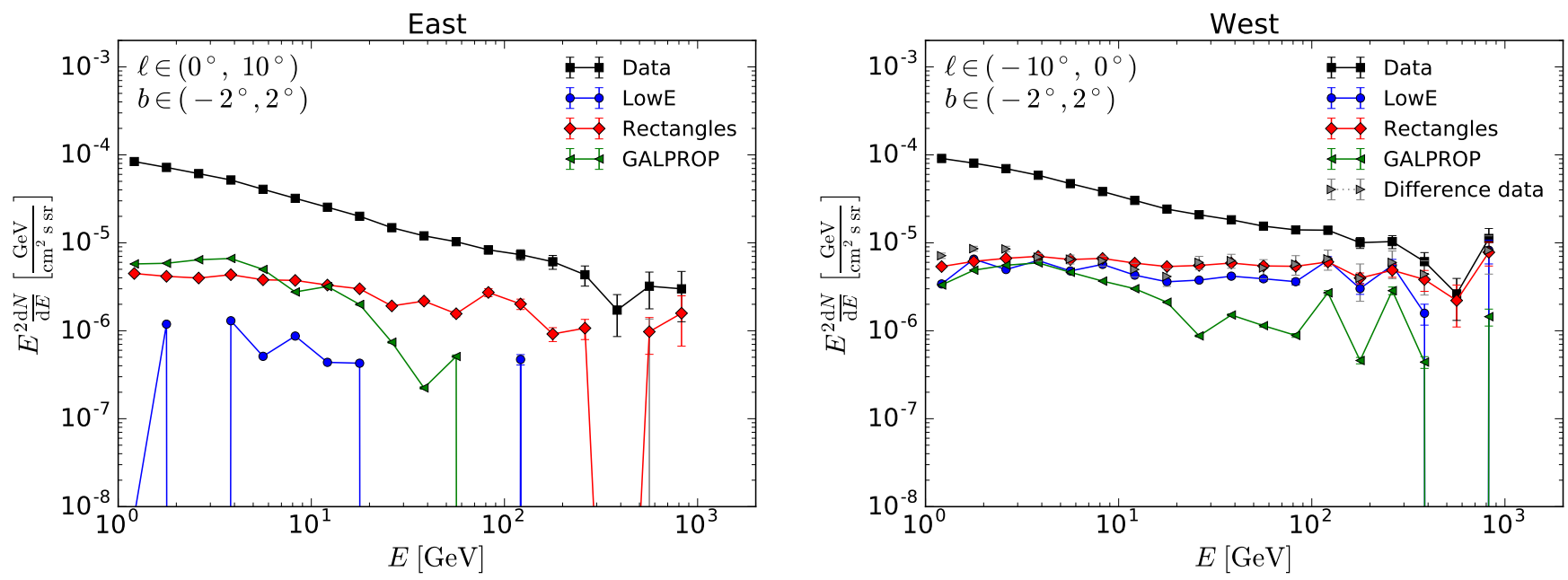

Fig. 9. Gamma-ray data SEDs of the residuals (LowE model), rectangles model of the FBs (rectangles model), residuals plus FBs model plus gNFW DM annihilation template (GALPROP model), and of the difference in the data west minus east of the GC (excluding pixels in the PS mask).

base of the FBs. We associate the excess between $-8^{\circ} \lesssim \ell \lesssim-2^{\circ}$ to the base of the FBs. In this paper, we use $\ell \in\left(0^{\circ},-10^{\circ}\right)$ and $|b|<6^{\circ}$ in the calculations of the spectrum of the gamma-ray emission at the base of the FBs.

\subsection{Comparison of the spectra at different latitudes}

In this section we quantify the hardening of the gamma-ray spectrum at the base of the FBs. We first compare the spectral energy distribution (SED) of the emission at the base of the FBs for different foreground models in Fig. 9. The differential flux is averaged over regions to the east, $\ell \in\left(0^{\circ}, 10^{\circ}\right)$, and to the west, $\ell \in\left(-10^{\circ}, 0^{\circ}\right)$, of the GC in a thin stripe covering the GP $b \in\left(-2^{\circ}, 2^{\circ}\right)$. For comparison, we show the total data (excluding pixels masked by the PS mask) and, on the plot for $\ell \in\left(-10^{\circ}, 0^{\circ}\right)$, the difference in the data west minus east of the GC.

For negative longitudes, all models give similar results. The differential flux of the GALPROP model is smaller than the differential flux of the other models above $10 \mathrm{GeV}$, which is consistent with the profile plots in Fig. 7. The difference of the data west minus east of the GC is similar to the fluxes at the base of the FBs in the low-energy model and in the rectangles model. The spectra at positive longitudes show large oversubtractions and softer spectra.

To compare the behavior of the energy spectra at high energies for different latitudes, we fit a log-parabola plus the fixed foreground model counts to the total smoothed gamma-ray counts in each latitude stripe using the likelihood based on the gamma distribution. We use the following parametrization of the log-parabola function:

$f(E)=N_{0}\left(\frac{E}{1 \mathrm{GeV}}\right)^{-\alpha-\beta \ln (E / 1 \mathrm{GeV})}$.

The local "index" of the spectrum at energy $E$ is

$n \equiv-\frac{\mathrm{d} \ln f}{\mathrm{~d} \ln E}=\alpha+2 \beta \ln \left(\frac{E}{1 \mathrm{GeV}}\right)$.

In Fig. 10 we show this log-parabola index $n$ as a function of latitude at $E=500 \mathrm{GeV}$. We plot $(2-n)$, which corresponds to the SED index. For positive longitudes, the index is relatively 

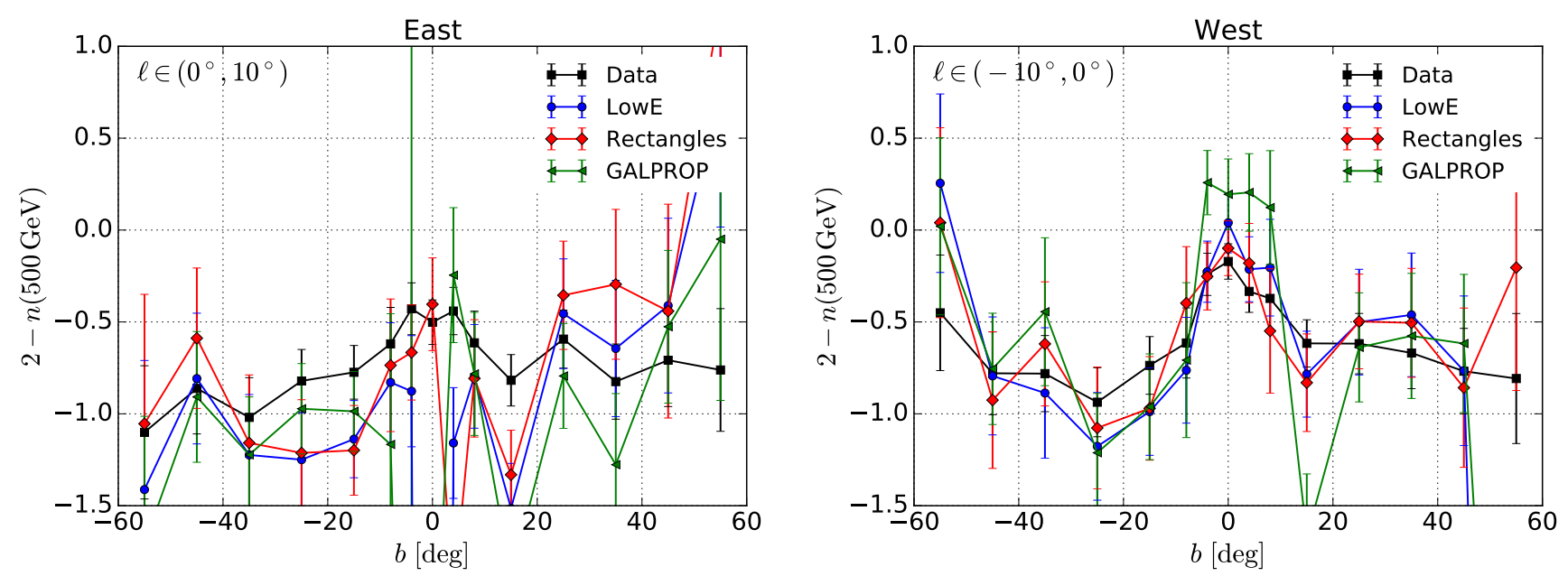

Fig. 10. Index of the log-parabola defined in Eq. (5) at $E=500 \mathrm{GeV}$ as a function of latitude for the FBs in the different foreground diffuse emission models.

soft $(n>2)$ for most of the latitudes except high latitudes where the gamma-ray statistics are small. For negative longitudes (i.e., west), the index near the GC is significantly harder $(n \approx 2)$ than the index at higher latitudes.

\subsection{Parametric model of the gamma-ray spectrum at low latitudes}

In this section we study the spectrum at the base of the FBs at latitudes $|b|<6^{\circ}$. As a baseline model we use the rectangles model of the FBs. We compare a power-law model of the energy spectrum with a power-law and an exponential cutoff model

$\frac{\mathrm{d} N}{\mathrm{~d} E}=N_{0}\left(\frac{E}{1 \mathrm{GeV}}\right)^{-n} e^{-E / E_{\mathrm{cut}}}$.

We fit the spectral model added to the fixed background to the total gamma-ray data counts. The best-fit parameters for the rectangles model are reported in Table 1 . If the improvement in $2 \Delta \log \mathcal{L}$ is less than 1 , then we report the power-law model parameters; it turns out that the improvement in $2 \Delta \log \mathcal{L}$ is less than 0.1 in the cases when the cutoff is not significant. For the model with a cutoff, we also find the $95 \%$ statistical confidence lower limit for the cutoff energy. In the last column we report the minimum among the $95 \%$ confidence lower limits for the three models considered in the paper (the low-energy data model, the rectangles model, and the GALPROP templates model). The spectrum of the residuals including the FBs at $\ell \in$ $\left(-10^{\circ}, 0^{\circ}\right)$ is consistent with a power law with an index 2.1-2.2 up to $E \approx 1 \mathrm{TeV}$. The $95 \%$ confidence lower limit on the cutoff energy is $\sim 500 \mathrm{GeV}$ for latitudes $|b|<6^{\circ}$ within $-10^{\circ}<\ell<0^{\circ}$.

As seen from the spectra in Fig. 9, there are large (relative to statistical uncertainty) fluctuations in some of the models. These fluctuations are due to modeling uncertainty of the Galactic diffuse emission. As a result, a fit with a simple function may be sensitive to the starting point (there can be several local minima). In the GALPROP model of the foreground, we also had to constrain the range of the index of the spectrum of the FB between 1.5 and 2.5 in the derivation of the $95 \%$ confidence lower limit on the cutoff energy.

\subsection{Inverse-Compton model of the gamma-ray emission}

In this section we model the gamma-ray emission at the base of the FBs with an IC scattering model. The SED of the gamma-ray intensity is parametrized as

$E_{\gamma}^{2} \frac{\mathrm{d} F_{\gamma}}{\mathrm{d} E_{\gamma}}=\frac{E_{\gamma} c}{4 \pi} \int \frac{\mathrm{d} n_{h v}}{\mathrm{~d} E_{h v}} \sigma_{\mathrm{IC}} \frac{\mathrm{d} \Sigma_{\mathrm{e}}}{\mathrm{d} E_{\mathrm{e}}} \mathrm{d} E_{h v} \mathrm{~d} E_{\mathrm{e}}$,

where $\frac{\mathrm{d} \Sigma_{\mathrm{e}}}{\mathrm{d} E_{\mathrm{e}}}=\int \frac{\mathrm{d} n_{\mathrm{e}}}{\mathrm{d} E_{\mathrm{e}}} \mathrm{d} R$ is the column density of CR electrons integrated along the line-of-sight distance $R, \frac{\mathrm{d} n_{h v}}{\mathrm{~d} E_{h v}}$ is the number density of ISRF photons of energy $h v$, and $\sigma_{\mathrm{IC}}\left(E_{\gamma}, E_{h v}, E_{\mathrm{e}}\right)$ is the differential IC scattering cross section in units of $E_{\gamma} \frac{\mathrm{d} \sigma}{\mathrm{d} E_{\gamma}}$ (Blumenthal \& Gould 1970). For details on the parametrization of $\sigma_{\text {IC }}$ see Appendix B of Ackermann et al. (2014). The ISRF number density for SL and IR photons is taken from Porter et al. (2008), which is available with the distribution of GALPROP v54.1, assuming that the emission at the base of the FBs is near the GC. For the CMB, we use the thermal spectrum with a temperature of $2.73 \mathrm{~K}$. There is a significant uncertainty in the Galactic ISRF energy density near the GC (e.g., Popescu et al. 2017; Porter et al. 2017; Niederwanger et al. 2019). We find (Appendix C) that this uncertainty can lead to a factor $\sim 3$ uncertainty in the overall normalization of the inferred CR electrons (CRe) energy density. Additional uncertainty on the CRe distribution and the associated IC emission comes from the distribution of the Galactic magnetic field (e.g., Orlando 2018, 2019). We model the column density of electrons as a power law with a cutoff

$\frac{\mathrm{d} \Sigma_{\mathrm{e}}}{\mathrm{d} E_{\mathrm{e}}}=n_{\mathrm{e}}\left(\frac{E_{\mathrm{e}}}{1 \mathrm{GeV}}\right)^{-\gamma_{\mathrm{e}}} e^{-E_{\mathrm{e}} / E_{\mathrm{cut}}}$.

In order to determine the normalization $n_{\mathrm{e}}$, the spectral index $\gamma_{\mathrm{e}}$, and the cutoff $E_{\text {cut }}$, we fit the IC model of the FBs plus the (fixed) foreground model counts to the total smoothed gammaray counts in different latitude stripes using likelihood based on the gamma distribution. As a baseline case, we take the gammaray spectrum derived in the rectangles model of the FBs in Sect. 3.3. The best-fit spectra for the rectangles model of the bubbles are shown in Fig. 11 in latitude stripes $b \in\left(2^{\circ}, 6^{\circ}\right)$, $b \in\left(-2^{\circ}, 2^{\circ}\right)$ and $b \in\left(-6^{\circ},-2^{\circ}\right)$. If the improvement in $2 \Delta \log \mathcal{L}$ with and without the cutoff is less than 0.1 , then we show only the parameters for the power-law model without a cutoff. For example, for negative longitudes the cutoff is not significant.

The $95 \%$ statistical lower limit on the cutoff energy for the rectangles model and the minimum among the three models 
L. Herold and D. Malyshev: Hard and bright gamma-ray emission at the base of the Fermi bubbles

Table 1. Best-fit parameters of the gamma-ray spectrum and the significance of the cutoff for the rectangles model of the FBs.

\begin{tabular}{cccccccc}
\hline \hline Lat & Lon & $\begin{array}{c}N_{0} \\
10^{-6} \mathrm{GeV}^{-1} \mathrm{~cm}^{-2} \mathrm{~s}^{-1} \mathrm{sr}^{-1}\end{array}$ & $n$ & $\begin{array}{c}E_{\text {cut }} \\
\mathrm{GeV}\end{array}$ & $\begin{array}{c}2 \Delta \log \mathcal{L} \\
E_{\text {cut,95\% }} \\
\mathrm{GeV}\end{array}$ & $\begin{array}{c}E_{\text {cut }, 95 \%}^{\text {min }} \\
\mathrm{GeV}\end{array}$ \\
\hline$\left(2^{\circ}, 6^{\circ}\right)$ & $\left(0^{\circ}, 10^{\circ}\right)$ & $1.5 \pm 0.3$ & $1.9 \pm 0.1$ & $45 \pm 17$ & 4.8 & 25 & 25 \\
& $\left(-10^{\circ}, 0^{\circ}\right)$ & $3.1 \pm 0.4$ & $2.2 \pm 0.05$ & - & $<0.1$ & 510 & 510 \\
$\left(-2^{\circ}, 2^{\circ}\right)$ & $\left(0^{\circ}, 10^{\circ}\right)$ & $6.4 \pm 1.1$ & $2.3 \pm 0.09$ & - & $<0.1$ & 300 & 2.4 \\
& $\left(-10^{\circ}, 0^{\circ}\right)$ & $7.9 \pm 0.7$ & $2.1 \pm 0.04$ & - & $<0.1$ & 1000 & 600 \\
$\left(-6^{\circ},-2^{\circ}\right)$ & $\left(0^{\circ}, 10^{\circ}\right)$ & $2.5 \pm 0.4$ & $2.1 \pm 0.07$ & $260 \pm 150$ & 3.8 & 130 & 2.3 \\
& $\left(-10^{\circ}, 0^{\circ}\right)$ & $4.0 \pm 0.3$ & $2.2 \pm 0.04$ & - & $<0.1$ & 560 & 560 \\
\hline
\end{tabular}

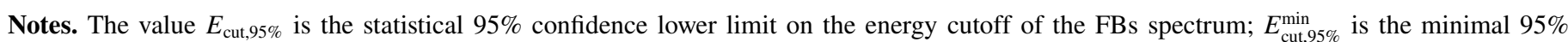
statistical lower limit among the models of the FBs in Sects. 3.2-3.4. There are four cases in which the introduction of a cutoff does not lead to improved fit: in all of these cases $2 \Delta \log \mathcal{L}<0.1$ while the formal best-fit values of $E_{\text {cut }}$ are larger than $1 \mathrm{TeV}$.

presented in Sects. 3.2-3.4 of the 95\% confidence lower limits for the cutoff energy are presented in Table 2. For negative longitudes, the $95 \%$ confidence lower limit on the cutoff in the spectrum of electrons in the rectangles model is about $4 \mathrm{TeV}$, while the minimal value of the $95 \%$ confidence limit for the three models of the foreground emission is about $3 \mathrm{TeV}$.

\subsection{Hadronic model of gamma-ray emission}

In the hadronic model, the gamma rays are produced as a result of collisions of hadronic CRs with the interstellar gas. The gammaray intensity in the hadronic model is parametrized as

$E_{\gamma}^{2} \frac{\mathrm{d} F_{\gamma}}{\mathrm{d} E_{\gamma}}=\frac{E_{\gamma}}{4 \pi} \int n_{\mathrm{H}} \sigma_{\mathrm{p}} v_{\mathrm{p}}\left(\frac{\mathrm{d} \Sigma}{\mathrm{d} T}\right)_{\mathrm{p}} \mathrm{d} T_{\mathrm{p}}$,

where $\left(\frac{\mathrm{d} \Sigma}{\mathrm{d} T}\right)_{\mathrm{p}}=\int\left(\frac{\mathrm{d} n}{\mathrm{~d} T}\right)_{\mathrm{p}} \mathrm{d} R$ is the column density of CR protons, $v_{\mathrm{p}}$ is the velocity of the protons, $T_{\mathrm{p}}=\sqrt{(q c)^{2}+\left(m_{\mathrm{p}} c^{2}\right)^{2}}-m_{\mathrm{p}} c^{2}$ is the kinetic energy of the protons as a function of momentum $q, \sigma_{\mathrm{p}}\left(E_{\gamma}, T_{\mathrm{p}}\right)$ is the differential cross section in units of $E_{\gamma} \frac{\mathrm{d} \sigma}{\mathrm{d} E_{\gamma}}$ for gamma rays in proton-proton collisions (Kamae et al. 2006; Karlsson \& Kamae 2008), and $n_{\mathrm{H}}$ is the density of gas. We use $n_{\mathrm{H}}=1 \mathrm{~cm}^{-3}$ as a reference density, which is comparable with the gas surface density of $\sim 30 M_{\odot} \mathrm{pc}^{-2}$ (Miville-Deschênes et al. 2017) averaged over the approximate height of the enhanced emission at the base of the bubbles, i.e., $\sim 900 \mathrm{pc}$ above and below the GC. Since most of the gas density is concentrated within 100-200 pc from the midplane (e.g., Sofue 2013; Miville-Deschênes et al. 2017), the gas density in the plane near the GC is $5-10 \mathrm{~cm}^{-3}$, which is consistent with the enhancement of the intensity of the emission at the base of the FBs at $\ell=0^{\circ}$. We model the proton spectrum as a power law of the momentum $\frac{\mathrm{d} \Sigma}{\mathrm{d} q c}=n_{\mathrm{p}} q^{-\gamma}$; ; we note that $v_{\mathrm{p}} \frac{\mathrm{d} \Sigma}{\mathrm{d} T_{\mathrm{p}}}=c \frac{\mathrm{d} \Sigma}{\mathrm{d} q c}$.

We fit the hadronic model of the gamma-ray emission at the base of the FBs analogously to the leptonic model in Sect. 4.4. The dash-dotted line in Fig. 11 represents the best-fit hadronic spectrum (labeled as $\pi^{0}$ ). The index of the proton spectrum is relatively hard $\gamma_{\mathrm{p}} \lesssim 2.3$, especially to the west of the GC.

We calculate the significance of a cutoff in the CR proton spectrum by adding an exponential cutoff factor and refitting the model to the gamma-ray data. The improvement in the model and the $95 \%$ confidence lower bound on the cutoff values are presented in Table 3 . Within $\pm 6^{\circ}$ from the GP west of the GC, the $95 \%$ confidence level for the lower bound on the cutoff among the models of the foreground emission that we have considered in Sects. 3.2-3.4 is about $6 \mathrm{TeV}$.

\subsection{Summary of the spectral analysis}

In Fig. 12 we show the envelopes of the gamma-ray spectra at $|b|<2^{\circ}$ of the emission at the base of the FBs for the models of foreground emission that we have considered, including the models in which we change the selection of the low energies in the definition of the foreground emission model (Appendix A). In order to determine the maximal and minimal models of the FBs in the GP, we fit the maximal and minimal points in the envelope above $3 \mathrm{GeV}$ with a power law with a cutoff function; we fit above $3 \mathrm{GeV}$ because some of the models of the foreground emission in Appendix A are determined for energies between 1 and $2.2 \mathrm{GeV}$. The corresponding parameters are reported in the first row of Table 4. We also fit the IC and hadronic models to the maximal and minimal points in the envelope and report the corresponding parameters in Table 4.

\section{Intepretation}

The bright and hard gamma-ray emission at the base of the bubbles can be at any position along the line of sight. In this section we consider two characteristic scenarios: that the emission is near the GC or that the emission is produced by one or a few supernova remnants (SNRs) closer to us.

\subsection{Emission near the GC scenario}

In the estimates in this subsection we use the following characteristic sizes: the distance to the GC is $8.5 \mathrm{kpc}$, the size of the region with the enhanced gamma-ray emission interpreted as an additional population of $\mathrm{CRs}$ is $-10^{\circ}<\ell<0^{\circ}$ and $|b|<6^{\circ}$. If we assume that the geometry of the region is a simple box, then the size of the box along the GP is $1.5 \mathrm{kpc}$, while the half-size in the vertical direction (distance from the GP to the boundary) is $\Delta h=0.9 \mathrm{kpc}$. The total gamma-ray luminosity in this case can be estimated by summing the data points in the rectangles model in Fig. 11 for $|b|<6^{\circ}$, which yields the luminosity of $L=1.1 \times 10^{37} \mathrm{erg} \mathrm{s}^{-1}$ above $1 \mathrm{GeV}$.

One of the most intriguing features of the gamma-ray spectrum at the base of the FBs is the absence of a significant cutoff up to $1 \mathrm{TeV}$ and an inferred hard spectrum of underlying electrons or protons. In particular, if the emission originates 

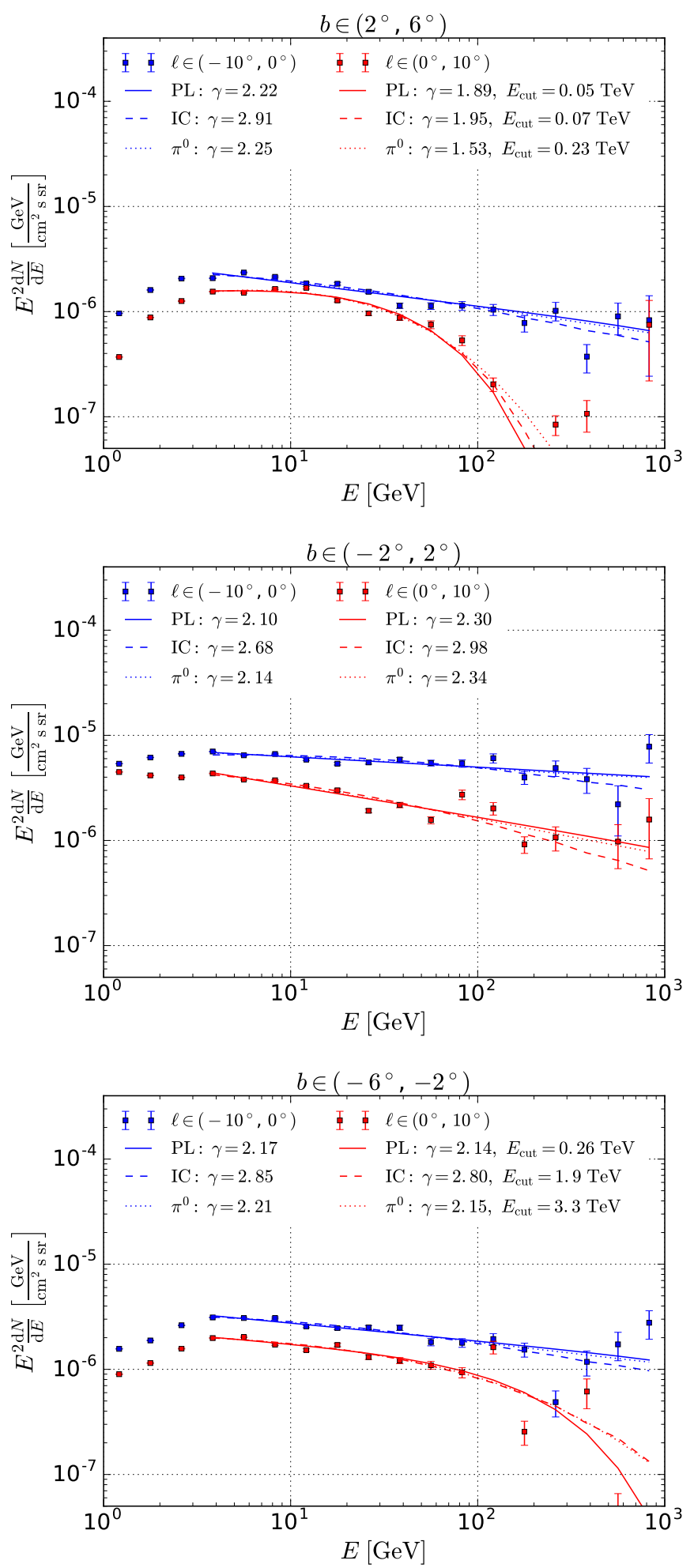

Fig. 11. Rectangles model SEDs of the FBs in the latitude stripes $\left(2^{\circ}, 6^{\circ}\right),\left(-2^{\circ}, 2^{\circ}\right)$ and $\left(-6^{\circ},-2^{\circ}\right)$ for negative (blue) and positive (red) longitudes. We compare the parametric model of the SED with the leptonic (Sect. 4.4) and hadronic (Sect. 4.5) models.

in hadronic interactions, the spectrum of the CR protons (CRp) is $\sim E^{-2.2}$, which is significantly harder than the propagated spectrum $\sim E^{-2.5-2.7}$ observed both locally (e.g., Casandjian 2015; Remy et al. 2017; Neronov et al. 2017; Prokhorov \& Colafrancesco 2018) and throughout the GP (e.g., Gaggero et al. 2015; Acero et al. 2016; Yang et al. 2016; Aharonian et al. 2018).
Table 2. Energy cutoff values and the significance of the cutoff in the IC model of the FBs at low latitudes.

\begin{tabular}{ccccc}
\hline \hline Lat & Lon & & \multicolumn{2}{c}{ Lower bound on $E_{\text {cut }}(\mathrm{TeV})$} \\
\cline { 4 - 5 }$\left(2^{\circ}, 6^{\circ}\right)$ & $\left(0^{\circ}, 10^{\circ}\right)$ & 2.6 & 0.20 & 0.17 \\
& $\left(-10^{\circ}, 0^{\circ}\right)$ & $<0.1$ & 4.0 & 4.0 \\
$\left(-2^{\circ}, 2^{\circ}\right)$ & $\left(0^{\circ}, 10^{\circ}\right)$ & $<0.1$ & 1.4 & 0.01 \\
& $\left(-10^{\circ}, 0^{\circ}\right)$ & $<0.1$ & 13 & 2.9 \\
$\left(-6^{\circ},-2^{\circ}\right)$ & $\left(0^{\circ}, 10^{\circ}\right)$ & 1.1 & 0.82 & 0.03 \\
& $\left(-10^{\circ}, 0^{\circ}\right)$ & $<0.1$ & 7.0 & 7.0 \\
\hline
\end{tabular}

Notes. The lower bounds for $E_{\text {cut }}$ at the $95 \%$ confidence level for our baseline model and the minimum among the models presented in Sects. 3.2-3.4 are shown in the last two columns, respectively.

Table 3. Energy cutoff values and the significance of the cutoff in the hadronic model of the FBs at low latitudes.

\begin{tabular}{ccccc}
\hline \hline & & & \multicolumn{2}{c}{ Lower bound on $E_{\text {cut }}(\mathrm{TeV})$} \\
\cline { 4 - 5 } Lat & Lon & $2 \Delta \log \mathcal{L}$ & Rectangles model & All models \\
\hline$\left(2^{\circ}, 6^{\circ}\right)$ & $\left(0^{\circ}, 10^{\circ}\right)$ & 4.4 & 0.88 & 0.48 \\
& $\left(-10^{\circ}, 0^{\circ}\right)$ & $<0.1$ & 7.4 & 7.4 \\
$\left(-2^{\circ}, 2^{\circ}\right)$ & $\left(0^{\circ}, 10^{\circ}\right)$ & $<0.1$ & 3.8 & 0.023 \\
& $\left(-10^{\circ}, 0^{\circ}\right)$ & $<0.1$ & 29 & 6.3 \\
$\left(-6^{\circ},-2^{\circ}\right)$ & $\left(0^{\circ}, 10^{\circ}\right)$ & 2.7 & 1.6 & 0.05 \\
& $\left(-10^{\circ}, 0^{\circ}\right)$ & $<0.1$ & 12 & 12 \\
\hline
\end{tabular}

Notes. The lower bounds for $E_{\text {cut }}$ at the $95 \%$ confidence level for our baseline model and the minimum among the models presented in Sects. 3.2-3.4 are shown in the last two columns, respectively.

Although there is a hardening of the spectrum of CRs in the inner rings of the Galaxy and near the GC, the inferred spectra of the CRs at the base of the FBs are significantly harder than the propagated spectra of the CRs in the inner rings and near the GC (Gaggero et al. 2015; Acero et al. 2016; Yang et al. 2016; Aharonian et al. 2018). We assume that the CRp spectrum at the base of the bubbles is equal to the injection spectrum unaffected by the propagation softening. This is possible if the CRp were injected relatively recently and had insufficient time to escape from the region of the enhanced emission, i.e., the age of the CRp is less than the propagation time to cross the vertical distance of $0.9 \mathrm{kpc}$. If we assume a spatially constant diffusion coefficient $D(E)=D_{0}\left(\frac{E}{1 \mathrm{GeV}}\right)^{\delta}$ with $D_{0}=$ $3 e 28 \mathrm{~cm}^{2} \mathrm{~s}^{-1}=100 \mathrm{pc}^{2} \mathrm{kyr}^{-1}$ and $\delta=0.4$ (Strong et al. 2007), then the escape time for the protons at $E>6 \mathrm{TeV}$ is

$T_{\mathrm{esc}}<\frac{\Delta h^{2}}{2 D(E)} \approx 100 \mathrm{kyr}$.

This gives us an approximate upper bound on the age of the proton CRs, assuming that the diffusion coefficient near the GC is similar to the diffusion coefficient near Sun. The escape time can be significantly longer if the diffusion length near the GC is smaller than the local diffusion length or if the CRs are confined by a particular configuration of magnetic fields. The CRp 

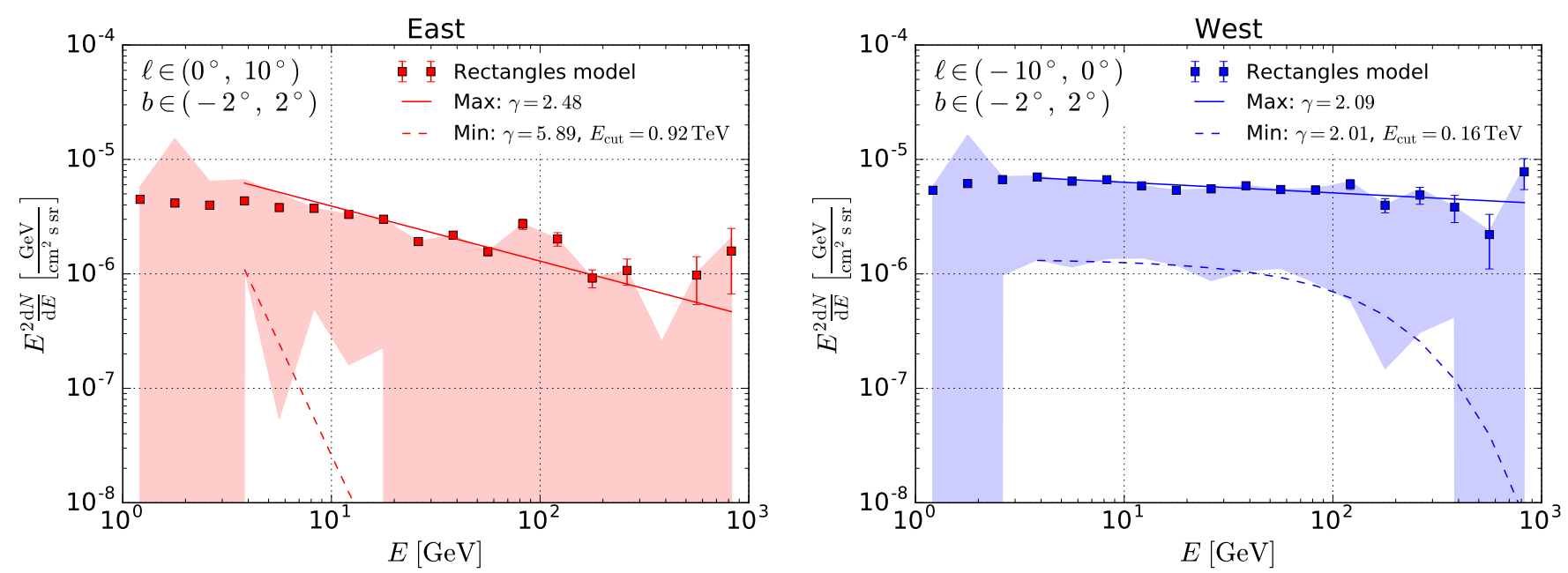

Fig. 12. Spectral energy distribution of the FBs in the GP. The shaded areas show the envelope of the spectra of the FBs in all foreground models considered in the paper including the changes in the choice of the low-energy range to model the foreground emission (discussed in Appendix A). The lines show the fits to the maximal and minimal points in the envelopes above $3 \mathrm{GeV}$.

Table 4. Summary of the minimal ("min") and maximal ("max") models for the parametric, IC, and hadronic models of the FBs for $|b|<2^{\circ}$ and $-10^{\circ}<\ell<0^{\circ}$.

\begin{tabular}{lccccc}
\hline Model & Type & Norm & $\begin{array}{c}\text { Index } \\
\text { Parametric, }, \frac{\mathrm{d} N_{\gamma}}{\mathrm{d} E}\left(\mathrm{GeV}^{-1} \mathrm{~cm}^{-2} \mathrm{~s}^{-1}\right)\end{array}$ & $\begin{array}{c}\text { Max } \\
\mathrm{TeV}\end{array}$ & $\begin{array}{c}E_{\text {cut,95\% }} \\
\mathrm{TeV}\end{array}$ \\
\hline Min & $1.4 \times 10^{-6}$ & 2.01 & 0.16 & 0.04 \\
$\mathrm{IC}, \frac{\mathrm{d} \Sigma_{e}}{\mathrm{~d} E}\left(\mathrm{GeV}^{-1} \mathrm{~cm}^{-2}\right)$ & Max & $3.4 \times 10^{10}$ & 2.67 & - & 19 \\
Hadronic, $\frac{\mathrm{d} \Sigma_{p}}{\mathrm{~d} q c}\left(\mathrm{GeV}^{-1} \mathrm{~cm}^{-2}\right)$ & Min & $1.3 \times 10^{10}$ & 2.81 & 7.2 & 0.96 \\
& Max & $9.5 \times 10^{11}$ & 2.13 & - & 65 \\
& Min & $1.1 \times 10^{11}$ & 1.98 & 1.8 & 0.23 \\
\hline
\end{tabular}

Notes. For the parametric model, we report the energy spectrum of the gamma rays, for the IC model we report the column density of the spectrum of the electrons as a function of energy, while for the hadronic model we list the column density of the spectrum of the protons as a function of momentum. The spectra are normalized at $E_{0}=1 \mathrm{GeV}$. The last column shows the statistical $95 \%$ confidence lower limit on the cutoff, $E_{\mathrm{cut}, 95 \%}$.

energy density within $|b|<6^{\circ}$ in the rectangles model of the FBs (Sect. 4.5) normalized to the reference density of $n_{\mathrm{H}}=1 \mathrm{~cm}^{-3}$ is $\mathrm{d} E_{\mathrm{tot}} / \mathrm{d} V=360 \mathrm{meV} \mathrm{cm}^{-3}$ above $1 \mathrm{GeV}$. In Fig. 13 (left), we compare the corresponding flux to the local CRp flux. The total energy in CRp within $|b|<6^{\circ}$ is $E_{\text {tot }}=7 e 52 \mathrm{erg}$. This population of $\mathrm{CRp}$ can be obtained from $\sim 700 \mathrm{SNe}$, assuming that on average $\mathrm{SNe}$ inject $\sim 10^{50} \mathrm{erg}$ in $\mathrm{CRp}$, which corresponds to about $10 \%$ efficiency of CR acceleration by a SN with kinetic energy $\sim 10^{51}$ erg (e.g., Spurio 2015). There are several populations of young stellar objects near the GC (Yusef-Zadeh et al. 2009; Immer et al. 2012); in particular there is a population of stars close to the GC with an age of about $6 \mathrm{Myr}$ (Paumard et al. 2006). If we assume that the rate of SNe in the Galaxy is about one per century and that the GC region contributes at the level of a few percent to the total rate, for example a few SNRs per $10 \mathrm{kyr}$, then the 700 SNRs can be produced near the GC in the past few million years.

The best-fit spectrum of CRe is $\sim E^{-2.7}$, which is softer than the spectrum of the protons. Nonetheless, this spectrum is harder than the spectrum expected in the presence of cooling for a stationary population of CRe. Thus, unless the injection spectrum is harder than $E^{-2}$, the population of the CRe at the base of the bubbles is not affected by cooling up to $3 \mathrm{TeV}$, which is the $95 \%$ confidence minimal lower bound on the cutoff in the CRe (Table 2). The cooling time for the electrons at $3 \mathrm{TeV}$ is $T_{\text {cool }} \approx 200 \mathrm{kyr}$, which is comparable to the diffusive escape time from the $\pm 900 \mathrm{pc}$ volume around the GP at $3 \mathrm{TeV}$. The cooling time can have a factor $\sim 3$ uncertainty due to the uncertainties in the ISRF near the GC (see Appendix C).

The energy density in electrons with energies above $E_{0}=$ $1 \mathrm{GeV}$, which is necessary to produce the gamma-ray emission in the rectangles model of the FBs (Sect. 4.4), is $4.0 \mathrm{meV} \mathrm{cm}^{-3}$. In Fig. 13 (right), we compare the corresponding flux to the local CRe flux. The total energy content of the ROI in electrons above $1 \mathrm{GeV}$ is $E_{\text {tot }}=3 e 51 \mathrm{erg}$, which corresponds to the CR energy output of $3000 \mathrm{SNe}$, assuming a $0.1 \%$ efficiency in converting the SNR kinetic energy to CRe energy. The ratio of the electron and proton acceleration efficiencies $K_{\mathrm{e} / \mathrm{p}}=10^{-2}$ assumed in this work is relatively optimistic, given that the ratio of efficiencies can be as low as $\sim 10^{-3}$ (e.g., Park et al. 2015). Similar efficiencies of $K_{\mathrm{e} / \mathrm{p}} \propto 10^{-2}$ can be expected in the acceleration of electrons and protons in jets from SMBHs (e.g., Ball et al. 2018). Since the escape time is comparable to the electron cooling time and the required number of $\mathrm{SNe}$ in the hadronic scenario is smaller than the number of $\mathrm{SNe}$ in the leptonic scenario (for the ratio of acceleration efficiencies $K_{\mathrm{e} / \mathrm{p}} \lesssim 10^{-2}$ ), we conclude 

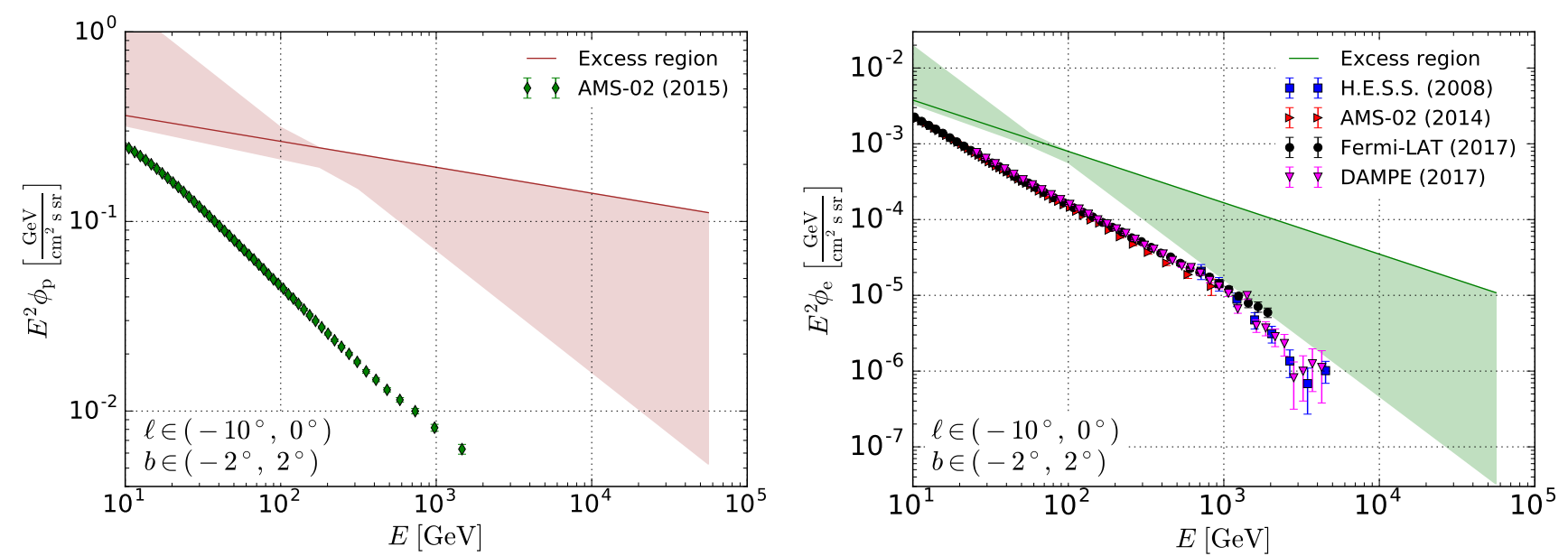

Fig. 13. Proton (left) and electron (right) spectra fitted to the gamma-ray emission at the base of the FBs. Solid lines show the best-fit spectra in the rectangles model of the FBs. The shaded bands represent the systematic uncertainties, estimated from the maximal and minimal best-fit FBs spectra among the foreground models. Uncertainties in the ISRF energy density near the GC result in an additional factor of $\sim 2$ uncertainties in the CRe energy density (see Appendix C for details). For comparison with the local CR spectrum, we show the local fluxes of CRp measured by AMS-02 (Aguilar et al. 2015) and CRe measured by H.E.S.S. (Aharonian et al. 2008), AMS-02 (Aguilar et al. 2014), Fermi-LAT (Abdollahi et al. 2017), and DAMPE (Ambrosi et al. 2017). The error bars represent the systematic and statistical uncertainties added in quadrature.
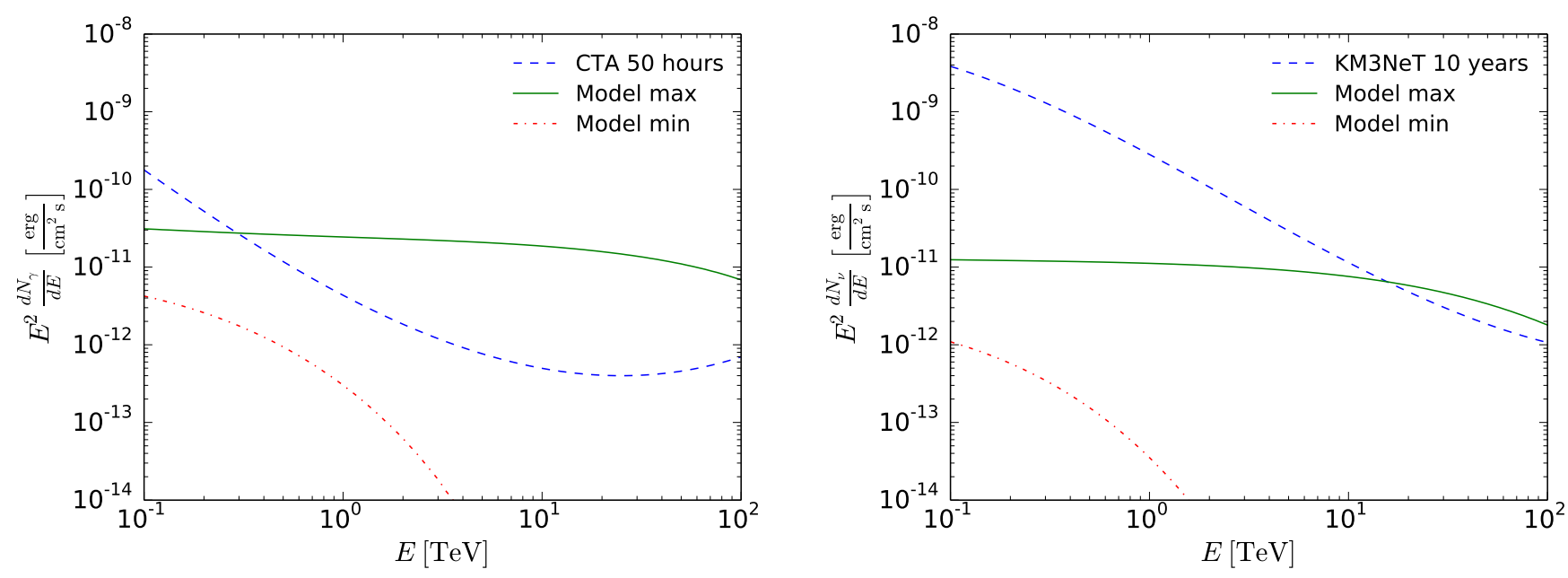

Fig. 14. Comparison of the hadronic model of the gamma-ray emission at the base of the FBs with CTA and KM 3 NeT for a source with $2^{\circ}$ radius (Ambrogi et al. 2018). The minimal (min) and maximal (max) models correspond to the minimal and maximal models in the hadronic scenario in Table 4 (for the maximal model we assume a cutoff in the proton spectrum at $1 \mathrm{PeV}$ ) integrated over a $2^{\circ}$ circle to transform intensity to flux. The KM3NeT sensitivity is for muon neutrinos only. In estimating the sensitivity, we take into account that the GC is below the horizon for about two-thirds of the time at the KM3NeT location in the Mediterranean Sea.

that the majority of the gamma-ray emission near the base of the FBs is likely to be produced by the hadronic interactions of CRp with the gas. The presence of the hadronic production of gamma rays at the base of the FBs, without any sign of a cutoff up to gamma-ray energies of $1 \mathrm{TeV}$, is important for the detectability of the associated neutrino signal by neutrino telescopes; see Fig. 14 (see also Razzaque \& Yang 2018).

\subsection{Nearby SNR or a superbubble scenario}

In this subsection we discuss models where the hard and bright gamma-ray emission at the base of the FBs is created either by a single SNR or several SNRs at a distance closer than the distance to the GC. We start with a single SNR scenario. For the leptonic model we use the local ISRF and calculate the IC emission as described in Sect. 4.4. Assuming one SNR, we find a distance of $50 \mathrm{pc}$. The radius of an SNR corresponding to $6^{\circ}$ circle at a distance of $50 \mathrm{pc}$ is about $5 \mathrm{pc}$. Provided that the
SNR would be rather close to Earth, it should be detected in radio and X-ray observations, however no suitable candidates are currently known (Green 2014, 2017). For the hadronic model of the gamma-ray emission, we assume the local gas density of $1 \mathrm{~cm}^{-3}$. At the GC, we need about 700 SNRs for this reference gas density; thus the distance at which a single SNR can explain the excess emission is $d=\frac{8.5 \mathrm{kpc}}{\sqrt{700}} \approx 300 \mathrm{pc}$. We can compare the flux from the low-latitude bubbles with the emission of a known SNR, such as Tycho's SNR. The differential energy flux of Tycho's SNR at $10 \mathrm{GeV}$ is $E^{2} \mathrm{~d} F / \mathrm{d} E \approx$ $10^{-6} \mathrm{MeV} \mathrm{cm}^{-2} \mathrm{~s}^{-1}$ (Archambault et al. 2017). The solid angle of the ROI of $10^{\circ} \times 12^{\circ}$ at the base of the FBs is $\Omega \approx 0.037$ sr. If we assume that Tycho's SNR is at a distance of $3 \mathrm{kpc}$, then the average intensity inside $\Omega$ at a distance of $300 \mathrm{pc}$ is $E^{2} \mathrm{~d} N / \mathrm{d} E \approx$ $2.7 \times 10^{-6} \mathrm{GeV} \mathrm{cm}^{-2} \mathrm{~s}^{-1} \mathrm{sr}^{-1}$, which is comparable to the FBs emission at latitudes $|b|<6^{\circ}$ (e.g., Fig. 12). The radius of Tycho's SNR is about $3.5 \mathrm{pc}$ (for the angular diameter of $8^{\prime}$ at $3 \mathrm{kpc}$ ), 


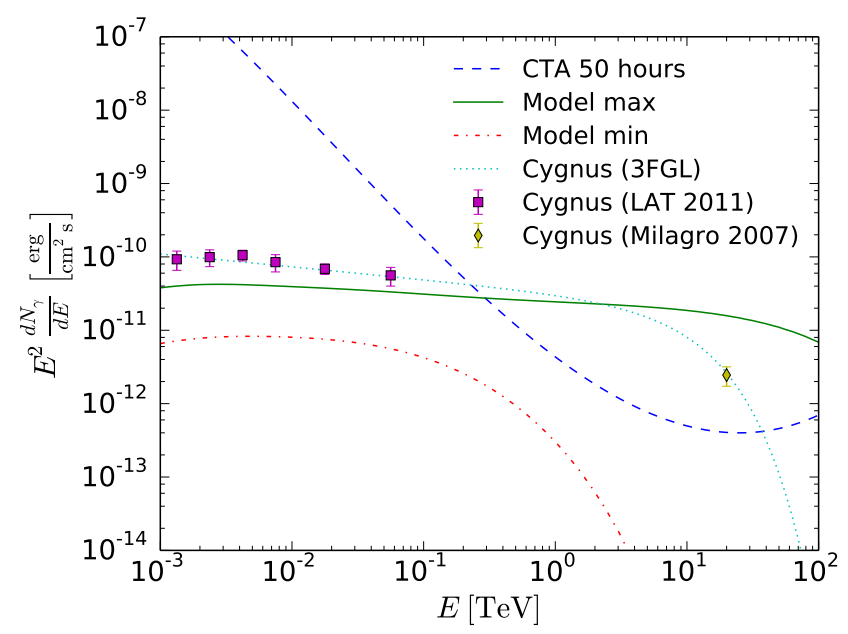

Fig. 15. Comparison of the minimal and maximal models of the residual flux at the base of the FBs with the Cygnus cocoon spectrum. The minimal and the maximal models are the same as in Fig. 14. For the 3FGL spectrum we take the power-law parameters from the catalog (Acero et al. 2015) and add a cutoff in the gamma-ray spectrum at $10 \mathrm{TeV}$ so that the SED is consistent with the Milagro source MGRO $\mathrm{J} 2031+41$ at $20 \mathrm{TeV}$ (Abdo et al. 2007). The purple squares represent the measurement of the flux from the Cygnus cocoon by the Fermi-LAT collaboration (Ackermann et al. 2011).

while the radius corresponding to $6^{\circ}$ angular size at $300 \mathrm{pc}$ is about $30 \mathrm{pc}$, which is almost ten times larger than the radius of Tycho's SNR. If we assume that only the core of the emission at the base of the FBs within $|b|<2^{\circ}$ corresponds to an SNR, the corresponding linear size is $\approx 10 \mathrm{pc}$ (for the same distance of $300 \mathrm{pc}$ ). There are several SNRs in Green's catalog (Green 2014,2017 ) between $-10^{\circ}<\ell<0^{\circ}$, but all of these have relatively small angular diameters $\lesssim 30^{\prime}$. Thus a single SNR is not a plausible scenario for the emission at the base of the FBs, unless this SNR is missing in Green's catalog.

Another possibility is that the emission at the base of the FBs is created by several SNRs and/or wind from massive stars, similar to the Cygnus cocoon (Ackermann et al. 2011) or the Loop I and the local superbubbles (Smith \& Cox 2001; Breitschwerdt \& de Avillez 2006; Wolleben 2007; Breitschwerdt et al. 2016; Schulreich et al. 2018; Dickinson 2018; Shchekinov 2018). The Cygnus cocoon (Ackermann et al. 2011) has a radius of about $2^{\circ}$, which at a distance of $1.4 \mathrm{kpc}$ corresponds to a radius of about $50 \mathrm{pc}$. If a Cygnus-like region is responsible for the emission within $|b|<6^{\circ}$ at the base of the FBs, then such region should be at a distance of about $500 \mathrm{pc}$. If only the core of the high-intensity region within $|b|<2^{\circ}$ is explained by such a cocoon, then the distance would be similar to the distance to the Cygnus cocoon, i.e., about 1.4 kpc. In Fig. 15 we compare the gamma-ray intensity at the base of the FB integrated over a $2^{\circ}$ circle to the flux from the Cygnus cocoon (Abdo et al. 2007; Ackermann et al. 2011). The flux in the maximal model is comparable (within a factor of 2) to the flux from the Cygnus cocoon between 1 and $100 \mathrm{GeV}$.

Massive stars, which can inflate cocoons or superbubbles by stellar winds and SN explosions, are usually found in young open stellar clusters. There are several young open stellar clusters in the direction of the base of the FBs, i.e., within $-10^{\circ}<\ell<0^{\circ}$. For example, clusters Trumpler 27 (Moffat et al. 1977) or NGC 6383 (Lloyd Evans 1978) contain more than ten massive OB stars, have an age of about $10 \mathrm{Myr}$, and an estimated distance of 1.2 and $1 \mathrm{kpc}$, respectively, in the direction of $\ell \approx 356^{\circ}$, $b \approx 0^{\circ}$. There is also a cluster NGC 6405 (Rohlfs et al. 1959), which has a smaller estimated distance of about $500 \mathrm{pc}$ but an older age $\sim 100 \mathrm{Myr}$. The turbulence created by winds from the massive stars and the past SNRs in these clusters can (re)accelerate CRs and it would also lead to smaller diffusion length, which would prevent the escape of CRs on timescales shorter than a few Myr (Ackermann et al. 2011). There are relatively few SNRs detected in gamma rays in that area of the sky. In particular, there is only one SNR, 3FGL J1741.1-3053, in the 3FGL catalog (Acero et al. 2015) that is between $-10^{\circ}<\ell<0^{\circ}$. This SNR is associated with the Tornado nebula at a distance of $12 \mathrm{kpc}$ (Castro et al. 2013). Green's catalog of SNRs contains several SNRs between $-10^{\circ}<\ell<0^{\circ}$, but SNRs with measured distances are more than $4 \mathrm{kpc}$ away. The absence of detected SNRs in the direction of the base of the FBs at distances $\lesssim 1.5$ kpc does not necessarily mean that there were no SNRs in the open clusters mentioned above. A possible reason is that the wind from the massive stars as well as the first SNRs in the cluster create a hot superbubble with smaller gas density than the average density in the GP. As a result, the subsequent SNRs quickly expand in the less dense environment until they reach the common envelope of the superbubble. For instance, this is observed in simulations of the formation of the local hot superbubble (Breitschwerdt et al. 2016; Schulreich et al. 2017, 2018). Whether the stellar clusters can provide the necessary CR injection rate to power the gamma-ray emission at the base of the FBs deserves a separate detailed study.

An interesting question is whether the FBs themselves can be inflated by a Cygnus-like superbubble, which accidentally happened along the line of sight towards the GC. If the FBs are at a distance of about $1 \mathrm{kpc}$, then their vertical size is also about $1 \mathrm{kpc}$, which is ten times smaller than the size of the FBs at a distance of $8.5 \mathrm{kpc}$. In the leptonic scenario of the FBs, the $10^{52} \mathrm{erg}$ in CRe at $8.5 \mathrm{kpc}$ (Ackermann et al. 2014) would correspond to $10^{50} \mathrm{erg}$ at $1 \mathrm{kpc}$. This power can be provided by several hundred $\mathrm{SNe}$, which is a relatively large number given the few young stellar clusters at distances $\lesssim 1 \mathrm{kpc}$ in the direction of the base of the FBs. This estimate is based on an assumed $0.1 \%$ efficiency in acceleration of CRe by SN shells; if there were an additional acceleration of the electrons by the turbulence either at the base of the FBs or inside the highlatitude FB volume, then the required number of SNRs would be smaller.

The electrons can be delivered to the FBs volume by an outflow that is possibly created by the pressure of the CRs themselves (e.g., Jacob et al. 2018). The measured red- and blueshift velocities of the gas outflow in the direction of the FBs are about 200-300 $\mathrm{km} \mathrm{s}^{-1}$, which implies the gas outflow from the GP in a biconical model of the FBs with velocities $\gtrsim 900 \mathrm{~km} \mathrm{~s}^{-1}$ and an age of the outflow at the position above the GC about 6-9 Myr (Fox et al. 2015; Bordoloi et al. 2017). If the distance is ten times smaller, then the age would be $\sim 600-900 \mathrm{kyr}$, which is comparable to the cooling time of $1 \mathrm{TeV}$ electrons necessary to explain the gamma-ray emission from the FBs at high latitudes (Ackermann et al. 2014). Thus the gamma-ray emission at high latitudes could be explained by an IC model with about 100 SNRs (or fewer if there is re-acceleration of electrons) created $\sim 1$ Myr ago, while the bright and hard emission at the base of the bubbles can be explained by the hadronic emission from about 10 SNRs. The actual age of the CRs is determined by the confinement within the superbubble and can be also on the order of $1 \mathrm{Myr}$ or more. In this scenario, the star-forming region, which can power the emission at the base of the FBs and, possibly, the FBs themselves can be situated in the Sagittarius arm at a distance of 1-1.5 kpc in the direction of the GC. 


\section{Conclusions}

In this paper we use nine years of Fermi-LAT Pass 8 Source class data to study the gamma-ray emission at the base of the FBs. We use multiple methods to construct the foreground diffuse gamma-ray components and to determine the properties of the residual gamma-ray emission at the base of the FBs. We confirm the earlier findings that the emission at the base of the FB has a higher intensity than the FBs emission at high latitudes. The spectrum at the base of the FBs is consistent with a single power law without a cutoff up to about $1 \mathrm{TeV}$. The emission is slightly displaced from the GC to negative longitudes, which favors a starburst scenario of the bubbles formation, unless there is a mechanism to shift the gamma-ray emission away from Sgr A* in the SMBH scenario.

The gamma-ray emission at the base of the FBs can be explained by either a hadronic or leptonic model of gamma-ray production with $\mathrm{CR}$ spectra consistent with a power law without a cutoff. The index of the CRe (CRp) spectrum is 2.6-2.9 (2.0-2.3). We derive the $95 \%$ confidence lower bound on the cutoff in the CR electron and proton spectra by selecting the lowest $95 \%$ statistical confidence value among the models of the foreground emission considered in Sects. 3.2-3.4. Within $|b|<6^{\circ}$ and $-10^{\circ}<\ell<0^{\circ}$, the $95 \%$ confidence lower bound on the exponential cutoff in the $\mathrm{CRe}(\mathrm{CRp})$ spectrum is about 3 (6) $\mathrm{TeV}$.

If the location of the residual emission is near the GC, then the total gamma-ray luminosity is $L \approx 10^{37} \mathrm{erg} \mathrm{s}^{-1}$ and the required energy of $\mathrm{CRe}(\mathrm{CRp})$ above $1 \mathrm{GeV}$ is $E_{\mathrm{e}}=3 e 51 \mathrm{erg}$ $\left(E_{\mathrm{p}}=7 e 52 \mathrm{erg}\right)$. Although the total required energy in CRe is smaller than in CRp, the efficiency of acceleration of hadronic CRs is expected to be significantly higher than for leptonic CRs. In particular, with $10 \% \mathrm{CRp}$ acceleration efficiency, about 700 SNRs are needed to explain the gamma-ray emission in the hadronic scenario near the GC, while with $0.1 \%$ CRe acceleration efficiency, the required number of SNRs is 3000. For the GC scenario, the estimated diffusive escape time and the electron cooling time is $\sim 100 \mathrm{kyr}$. In this case a relatively high SN rate of one per $\sim 100 \mathrm{yr}$ at the location of the gamma-ray emission is required. If the average ISRF energy density is a factor of $\sim 2$ lower or higher than the ISRF in the reference model (Appendix $\mathrm{C}$ ), then both the cooling time and the required energy density of CRe is a factor of $\sim 2$ higher or lower than in the reference model, which nevertheless requires the same SN rate of one per $\sim 100 \mathrm{yr}$.

If the residual gamma-ray emission is produced at a closer distance, for instance at about $1 \mathrm{kpc}$ by a Cygnus-like cocoon or a superbubble in the Sagittarius arm, then the required number of SNRs is about two orders of magnitude smaller. For the superbubble scenario, the characteristic linear size is 10 times smaller, which leads to 100 times shorter diffusion time of a few kyr. This is shorter than the lifetime of individual SNRs. Both in the GC and in the superbubble scenarios, the age of the CR population can be greater than the diffusion time if there is an additional confinement by the SN shells or the shell of a superbubble.

Apart from the question of the location of the emission at the base of the FBs, we can revisit the question of the location of the FBs themselves: the FBs could have been inflated by a superbubble about $1 \mathrm{Myr}$ ago at a distance of $1 \mathrm{kpc}$ from the Sun. The CRs in the past superbubble may create a wind with velocities up to $\sim 1000 \mathrm{~km} \mathrm{~s}^{-1}$, which is sufficient to inflate a $1 \mathrm{kpc}$ large bubble within $1 \mathrm{Myr}$ so that the CR electrons in the wind do not cool below $1 \mathrm{TeV}$ and can explain the gamma-ray emission from the FBs at high latitudes. The gamma-ray emission at low latitudes is then explained (most naturally) by a hadronic scenario from a more recent starburst episode at about the same location.

In the future, the bright and hard gamma-ray emission at the base of the FB should be detectable with CTA, or a version of the High-Altitude Water Cherenkov (HAWC) Gamma-Ray Observatory in the southern hemisphere (Mostafa et al. 2017; Assis et al. 2017; Razzaque \& Yang 2018). Observations with Cherenkov telescopes should detect or constrain the cutoff energy in the gamma-ray spectrum in multi-TeV regime. In the hadronic scenario, for a sufficiently high cutoff value of the CRp spectrum, for instance around $1 \mathrm{PeV}$, the corresponding neutrino emission could be detected with the future KM3NeT telescope.

Acknowledgements. The authors would like to thank Seth Digel, Anna Franckowiak, Stefan Funk, Gudlaugur Johannesson, Tsunefumi Mizuno, Troy Porter, Andrew Strong, and Luigi Tibaldo for valuable comments and suggestions. Also we would like to thank Cristina Popescu for providing us the ISRF data files in fits format. The Fermi-LAT Collaboration acknowledges generous ongoing support from a number of agencies and institutes that have supported both the development and the operation of the LAT as well as scientific data analysis. These include the National Aeronautics and Space Administration and the Department of Energy in the United States, the Commissariat à l'Energie Atomique and the Centre National de la Recherche Scientifique/Institut National de Physique Nucléaire et de Physique des Particules in France, the Agenzia Spaziale Italiana and the Istituto Nazionale di Fisica Nucleare in Italy, the Ministry of Education, Culture, Sports, Science and Technology (MEXT), High Energy Accelerator Research Organization (KEK) and Japan Aerospace Exploration Agency (JAXA) in Japan, and the K. A. Wallenberg Foundation, the Swedish Research Council, and the Swedish National Space Board in Sweden.

\section{References}

Abazajian, K. N., Canac, N., Horiuchi, S., \& Kaplinghat, M. 2014, Phys. Rev. D, 90,023526

Abdo, A. A., Allen, B., Berley, D., et al. 2007, ApJ, 664, L91

Abdo, A. A., Ackermann, M., Ajello, M., Allafort, A., et al. 2011a, ApJ, 734, 28 Abdo, A. A., Ackermann, M., Ajello, M., et al. 2011b, ApJ, 734, 116 Abdollahi, S., Ackermann, M., Ajello, M., et al. 2017, Phys. Rev. D, 95, 082007 Acero, F., Ackermann, M., Ajello, M., et al. 2015, ApJS, 218, 23 Acero, F., Ackermann, M., Ajello, M., et al. 2016, ApJS, 223, 26 Ackermann, M., Ajello, M., Allafort, A., et al. 2011, Science, 334, 1103 Ackermann, M., Albert, A., Atwood, W. B., et al. 2014, ApJ, 793, 64 Ackermann, M., Ajello, M., Albert, A., et al. 2016, Phys. Rev. D, 93, 082001 Ackermann, M., Ajello, M., Albert, A., et al. 2017, ApJ, 840, 43

Aguilar, M., Aisa, D., Alpat, B., et al. 2014, Phys. Rev. Lett., 113, 221102 Aguilar, M., Aisa, D., Alpat, B., et al. 2015, Phys. Rev. Lett., 114, 171103 Aharonian, F., Akhperjanian, A. G., Barres de Almeida, U., et al. 2008, Phys. Rev. Lett., 101, 261104

Aharonian, F., Peron, G., Yang, R., Casanova, S., \& Zanin, R. 2018, ArXiv e-prints [arXiv: 1811.12118]

Ambrogi, L., Celli, S., \& Aharonian, F. 2018, Astropart. Phys., 100, 69

Ambrosi, G., An, Q., Asfandiyarov, R., et al. 2017, Nature, 552, 63

Archambault, S., Archer, A., Benbow, W., et al. 2017, ApJ, 836, 23

Assis, P., Barres de Almeida, U., Blanco, A., et al. 2017, PoS ICRC2017 784

Ball, D., Sironi, L., \& Özel, F. 2018, ApJ, 862, 80

Blumenthal, G. R., \& Gould, R. J. 1970, Rev. Mod. Phys., 42, 237

Bordoloi, R., Fox, A. J., Lockman, F. J., et al. 2017, ApJ, 834, 191

Breitschwerdt, D., \& de Avillez, M. A. 2006, A\&A, 452, L1

Breitschwerdt, D., Feige, J., Schulreich, M. M., et al. 2016, Nature, 532, 73

Calore, F., Cholis, I., \& Weniger, C. 2015, J. Cosmol. Astropart. Phys., 3, 038

Casandjian, J.-M. 2015, ApJ, 806, 240

Castro, D., Slane, P., Carlton, A., \& Figueroa-Feliciano, E. 2013, ApJ, 774, 36

Dickinson, C. 2018, Galaxies, 6, 56

Fox, A. J., Bordoloi, R., Savage, B. D., et al. 2015, ApJ, 799, L7

Freudenreich, H. T. 1998, ApJ, 492, 495

Gaggero, D., Urbano, A., Valli, M., \& Ullio, P. 2015, Phys. Rev. D, 91, 083012

Goodenough, L., \& Hooper, D. 2009, ArXiv e-prints [arXiv:0910.2998]

Górski, K. M., Hivon, E., Banday, A. J., et al. 2005, ApJ, 622, 759

Green, D. A. 2014, Bull. Astron. Soc. India, 42, 47

Green, D. A. 2017, Cavendish Laboratory, Cambridge, United Kingdom (available at http://www.mrao.cam.ac.uk/surveys/snrs/)

Immer, K., Schuller, F., Omont, A., \& Menten, K. M. 2012, A\&A, 537, A121

Jacob, S., Pakmor, R., Simpson, C. M., Springel, V., \& Pfrommer, C. 2018, MNRAS, 475, 570 
L. Herold and D. Malyshev: Hard and bright gamma-ray emission at the base of the Fermi bubbles

Johannesson, G., Orlando, E., \& for the Fermi-LAT collaboration. 2013, ArXiv-eprints [arXiv:1307.0197]

Kamae, T., Karlsson, N., Mizuno, T., et al. 2006, ApJ, 647, 692

Karlsson, N., \& Kamae, T. 2008, ApJ, 674, 278

Lloyd Evans, T. 1978, MNRAS, 184, 661

Miville-Deschênes, M.-A., Murray, N., \& Lee, E. J. 2017, ApJ, 834, 57

Moffat, A. F. J., Fitzgerald, M. P., \& Jackson, P. D. 1977, ApJ, 215, 106

Moskalenko, I., \& Strong, A. 1998, ApJ, 493, 694

Mostafa, M., BenZvi, S., Schoorlemmer, H., Schüssler, F., et al. 2017, in APS April Meeting Abstracts, R4.005

Neronov, A., Malyshev, D., \& Semikoz, D. V. 2017, A\&A, 606, A22

Niederwanger, F., Reimer, O., Kissmann, R., et al. 2019, Astropart. Phys., 107, 1

Orlando, E. 2018, MNRAS, 475, 2724

Orlando, E. 2019, Phys. Rev. D, 99, 043007

Orlando, E., \& Strong, A. W. 2008, A\&A, 480, 847

Park, J., Caprioli, D., \& Spitkovsky, A. 2015, Phys. Rev. Lett., 114, 085003

Paumard, T., Genzel, R., Martins, F., et al. 2006, ApJ, 643, 1011

Popescu, C. C., Yang, R., Tuffs, R. J., et al. 2017, MNRAS, 470, 2539

Porter, T. A., Moskalenko, I. V., Strong, A. W., et al. 2008, ApJ, 682, 400

Porter, T. A., Jóhannesson, G., \& Moskalenko, I. V. 2017, ApJ, 846, 67

Prokhorov, D. A., \& Colafrancesco, S. 2018, MNRAS, 478, 2939

Ptuskin, V., Moskalenko, I. V., Jones, F., et al. 2006, ApJ, 642, 902

Razzaque, S., \& Yang, L. 2018, Galaxies, 6, 47

Remy, Q., Grenier, I. A., Marshall, D. J., \& Casandjian, J. M. 2017, A\&A, 601, A78
Robitaille, T. P., Churchwell, E., Benjamin, R. A., et al. 2012, A\&A, 545, A39

Rohlfs, K., Schrick, K. W., \& Stock, J. 1959, Z. Astrophys., 47, 15

Schulreich, M. M., Breitschwerdt, D., Feige, J., \& Dettbarn, C. 2017, A\&A, 604, A81

Schulreich, M., Breitschwerdt, D., Feige, J., \& Dettbarn, C. 2018, Galaxies, 6,26

Shchekinov, Y. 2018, Galaxies, 6, 62

Smith, R. K., \& Cox, D. P. 2001, ApJS, 134, 283

Sofue, Y. 2013, Mass Distribution and Rotation Curve in the Galaxy (Dordrecht: Springer Science+Business Media), eds. T. D. Oswalt \& G. Gilmore, 985

Spurio, M. 2015, Particles and Astrophysics: a Multi-Messenger Approach (Switzerland: Springer)

Storm, E., Weniger, C., \& Calore, F. 2017, J. Cosmol. Astropart. Phys., 8, 022

Strong, A. W., Moskalenko, I. V., \& Reimer, O. 2000, ApJ, 537, 763

Strong, A. W., Moskalenko, I. V., \& Reimer, O. 2004, ApJ, 613, 962

Strong, A. W., Moskalenko, I. V., \& Ptuskin, V. S. 2007, Ann. Rev. Nucl. Part. Sci., 57, 285

Su, M., \& Finkbeiner, D. P. 2012, ApJ, 753, 61

Su, M., Slatyer, T. R., \& Finkbeiner, D. P. 2010, ApJ, 724, 1044

Vladimirov, A. E., Digel, S. W., Johannesson, G., et al. 2011, Comput. Phys. Commun., 182, 1156

Wolleben, M. 2007, ApJ, 664, 349

Yang, R., Aharonian, F., \& Evoli, C. 2016, Phys. Rev. D, 93, 123007

Yusef-Zadeh, F., Hewitt, J. W., Arendt, R. G., et al. 2009, ApJ, 702, 178 


\section{Appendix A: Modeling and systematic uncertainties}
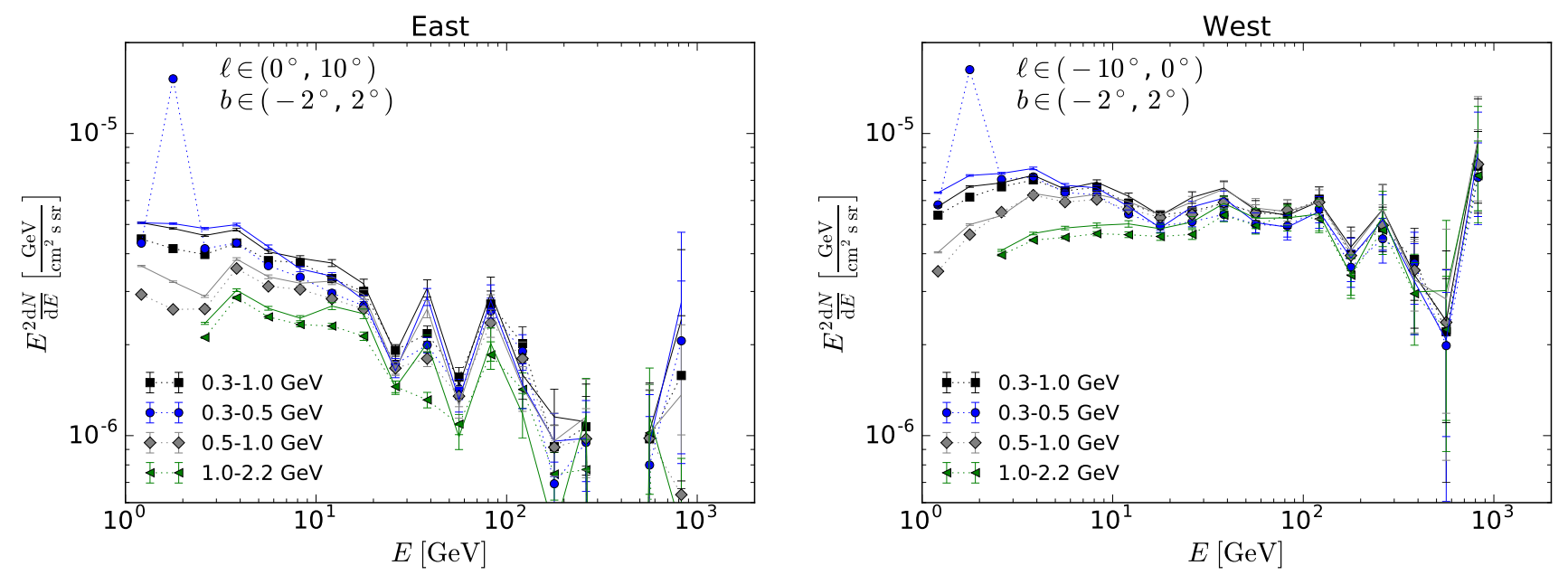

Fig. A.1. Residual SED in the rectangles model using Source class (dotted lines with markers) and UltraCleanVeto class data (solid lines) in four different energy ranges used to determine the foreground diffuse emission template. The baseline model, presented in Sect. 3.3, has the low-energy range in the definition of the foreground model $0.3-1.0 \mathrm{GeV}$ (black squares).

Table A.1. Best-fit parameters of the rectangles model without the last energy bin: $680 \mathrm{GeV}-1 \mathrm{TeV}$.

\begin{tabular}{cccccccc}
\hline \hline Lat & Lon & $\begin{array}{c}N_{0} \\
10^{-6} \mathrm{GeV}^{-1} \mathrm{~cm}^{-2} \mathrm{~s}^{-1} \mathrm{sr}^{-1}\end{array}$ & $n$ & $\begin{array}{c}E_{\text {cut }} \\
\mathrm{GeV}\end{array}$ & $2 \Delta \log \mathcal{L}$ & $\begin{array}{c}E_{\text {cut,95\% }} \\
\mathrm{GeV}\end{array}$ & $\begin{array}{c}E_{\text {cut } 95 \%}^{\min \%} \\
\mathrm{GeV}\end{array}$ \\
\hline$\left(2^{\circ}, 6^{\circ}\right)$ & $\left(0^{\circ}, 10^{\circ}\right)$ & $1.5 \pm 0.4$ & $1.9 \pm 0.2$ & $45 \pm 22$ & 6.8 & 25 & 25 \\
& $\left(-10^{\circ}, 0^{\circ}\right)$ & $3.0 \pm 0.4$ & $2.2 \pm 0.07$ & $720 \pm 1100$ & 0.45 & 210 & 210 \\
$\left(-2^{\circ}, 2^{\circ}\right)$ & $\left(0^{\circ}, 10^{\circ}\right)$ & $6.1 \pm 1.0$ & $2.3 \pm 0.08$ & $660 \pm 1000$ & 0.42 & 180 & 2.0 \\
& $\left(-10^{\circ}, 0^{\circ}\right)$ & $7.5 \pm 0.8$ & $2.1 \pm 0.04$ & $1400 \pm 1500$ & 0.76 & 490 & 460 \\
$\left(-6^{\circ},-2^{\circ}\right)$ & $\left(0^{\circ}, 10^{\circ}\right)$ & $2.5 \pm 0.3$ & $2.1 \pm 0.07$ & $260 \pm 160$ & 3.4 & 130 & 2.3 \\
& $\left(-10^{\circ}, 0^{\circ}\right)$ & $3.9 \pm 0.4$ & $2.1 \pm 0.05$ & $790 \pm 820$ & 0.96 & 290 & 290 \\
\hline
\end{tabular}

Notes. The definitions of the columns are the same as in Table 1.

In this appendix we study the dependence of the spectrum of gamma-ray emission at the base of the FB on the selection of the low-energy range in the definition of the diffuse foreground model and on the choice of the class of the events (UltraCleanVeto versus Source). We use the rectangles model of the FB in Sect. 3.3 as our baseline. In this model, the foreground emission template consists of Source data integrated over the energy interval $0.3-1.0 \mathrm{GeV}$. To probe the dependence on the choice of the low-energy range in the model, we pick three non-overlapping energy ranges, $0.3-0.5,0.5-1.0$, and $1.0-2.2 \mathrm{GeV}$, and use the same analysis as for the baseline model. We also compare with the spectra obtained using UltraCleanVeto data both in the definition of the model at low energies and in the analysis at high energies. The residual spectra resulting from the different energy ranges and data classes are shown in Fig. A.1. The agreement among the models is reasonably good at $E>10 \mathrm{GeV}$, especially for negative longitudes.

As shown in Fig. A.1, the highest energy spectral point for negative longitudes may have an upward statistical fluctuation, which can influence the conclusions about the lower limit in the cutoff values. In order to test the dependence on the highest energy spectral point, we exclude the energy bin $680 \mathrm{GeV}-1 \mathrm{TeV}$ and repeat the derivation of the best-fit parameters in the rectangles model of the FBs. The results are presented in Table A.1. Without the last data point, the four areas with infinite cutoff energy now have a finite cutoff, but with a significance $2 \Delta \log L<1$, i.e., the models are still consistent with a simple power law. The $95 \%$ confidence lower limit on the cutoff value is also smaller for the dataset without the last point, but in this case we are using the data up to $680 \mathrm{GeV}$ rather than $1 \mathrm{TeV}$.

\section{Appendix B: Gamma distribution as a likelihood for smoothed data}

In this appendix we show that smoothing the data and using gamma distribution instead of the Poisson distribution is a reasonable procedure, at least in the case when the scale of variations in the diffuse emission is larger than the smoothing radius. We split the log likelihood into a sum over areas with size approximately equal to the smoothing scale and neglect the correlation between the different areas after smoothing the data. We suppose that one of these areas has $n$ pixels with random counts $k_{i}$ drawn from a Poisson distribution with mean $\lambda_{0}$; in this case we assume that the underlying diffuse flux is approximately constant within the smoothing radius. The likelihood function for parameter $\lambda$ is

$L(\lambda)=\prod_{i} \frac{\lambda^{k_{i}}}{k_{i} !} e^{-\lambda}$. 
The log likelihood is

$\log L=\sum_{i=1}^{n}\left(-\lambda+k_{i} \log \lambda\right)+$ const.

The maximum likelihood value $\lambda_{*}$ is determined from

$0=\frac{\partial \log L}{\partial \lambda}=-n+\frac{1}{\lambda} \sum_{i=1}^{n} k_{i}$,

which gives $\lambda_{*}=\frac{1}{n} \sum_{i=1}^{n} k_{i}$. The uncertainty is

$\frac{1}{\sigma^{2}}=-\left.\frac{\partial^{2} \log L}{\partial \lambda^{2}}\right|_{\lambda=\lambda_{*}}=\frac{n}{\lambda_{*}}$,

which gives $\sigma^{2}=\lambda_{*} / n$.

If the smoothing radius is comparable to the size of the region, then we can approximate the values $k_{i}$ with the average in the region $\tilde{k}_{i}=\bar{k}=\frac{1}{n} \sum_{i=1}^{n} k_{i}$; we note that $\tilde{k}_{i}$ are not integers. We take the gamma distribution for the likelihood function

$\tilde{L}=\prod_{i} \frac{\lambda^{\tilde{k}_{i}}}{\Gamma\left(\tilde{k}_{i}+1\right)} e^{-\lambda}$.

The log likelihood is

$\log \tilde{L}=\sum_{i=1}^{n}\left(-\lambda+\tilde{k}_{i} \log \lambda\right)+$ const.

The maximum likelihood solution is the same as in the Poisson case $\tilde{\lambda}_{*}=\bar{k}=\lambda_{*}$. The uncertainty is also the same as in the Poisson case, i.e.,

$\frac{1}{\tilde{\sigma}^{2}}=-\left.\frac{\partial^{2} \log \tilde{L}}{\partial \lambda^{2}}\right|_{\lambda=\tilde{\lambda}_{*}}=\frac{n}{\tilde{\lambda}_{*}}=\frac{1}{\sigma^{2}}$.

Thus, smoothing the data and using the gamma distribution (in this case) gives the same result as using the original Poisson distribution, which shows that the procedure is well defined from the statistical point of view and it gives reasonable results.

\section{Appendix C: Modeling uncertainty of ISRF near the GC}

In this appendix we discuss the uncertainty of the IC model of the gamma-ray emission at the base of the FBs related to modeling of the ISRF near the GC. In order to estimate this uncertainty, we compare the ISRF model of Porter et al. (2008;

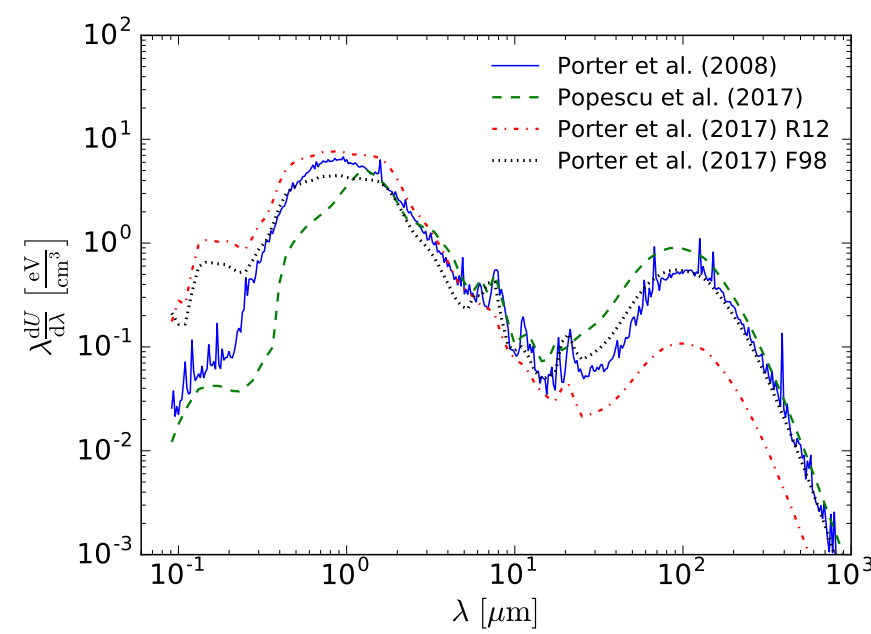

Fig. C.1. Comparison of ISRF SEDs at the GC (see text for more details).

available with the distribution of GALPROP v54.1), the ISRF model of Popescu et al. (2017), and the following two ISRF models of Porter et al. (2017): R12, based on Robitaille et al. (2012), and F98, based on Freudenreich (1998). In Fig. C.1 we show the corresponding densities of ISRFs averaged over the cylinder with the radius $R=1.5 \mathrm{kpc}$ and the height $z= \pm 0.3 \mathrm{kpc}$ around the GC, which approximately corresponds to the latitude stripe $|b|<2^{\circ}$ and $|\ell|<10^{\circ}$. We notice that there can be up to a factor of 2 differences in the ISRF energy density at the peak of the SL emission (around $1 \mu \mathrm{m}$ ) and an order of magnitude differences in the IR wavelengths (around $100 \mu \mathrm{m}$ ), which can affect the inferred populations of $\mathrm{CR}$ electrons producing the IC gamma rays (see also Porter et al. 2017; Niederwanger et al. 2019).

In Fig. C. 2 we show the IC models of the gamma-ray emission at the base of the FBs in the rectangle $b \in\left(-2^{\circ}, 2^{\circ}\right)$, $\ell \in\left(-10^{\circ}, 0^{\circ}\right)$ for the different ISRF models near the GC. We separate the SL, IR, and CMB contributions. The SL and IR ISRFs are separated by splitting the radiation field energy densities at $0.1 \mathrm{eV}(\approx 12 \mu \mathrm{m})$. We model the CR electrons spectra by a power-law function with an exponential cutoff. We fix the cutoff at $1 \mathrm{PeV}$ and fit the normalization and the spectral index by fitting the IC model to the Fermi-LAT spectral points. The corresponding parameters are presented in Table C.1. We use the Porter et al. (2008) ISRF as the reference model and show the normalizations of the CRe spectra relative to the reference model (the normalizations are determined at $1 \mathrm{GeV}$ ). The overall spread in the normalizations is about a factor of 3 , while the overall variation of the index of the CRe spectra is less than about 0.2 . 

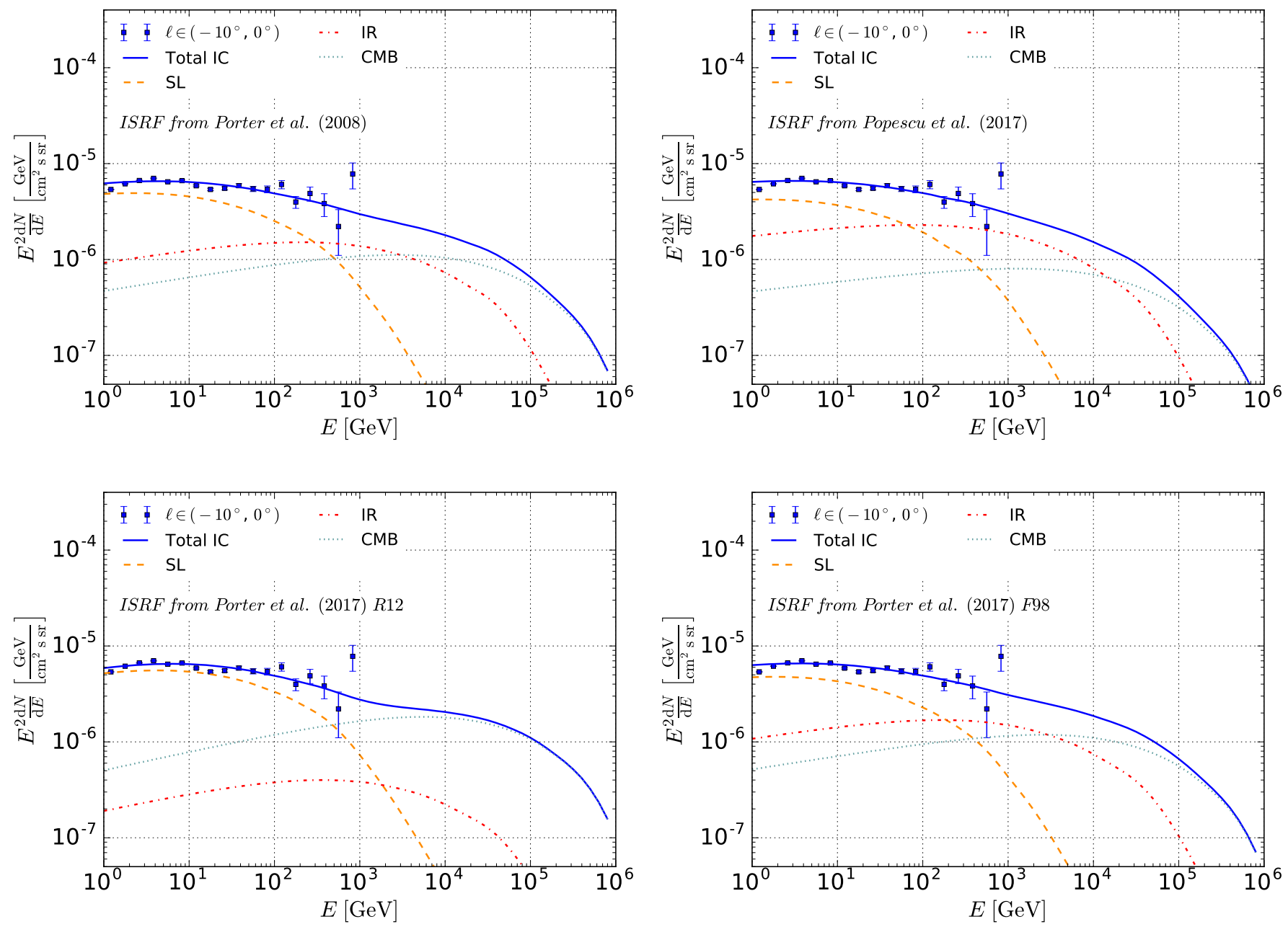

Fig. C.2. Contribution of various components of ISRFs to the IC model of the gamma-ray emission. The data points correspond to the emission at the base of the FBs in the rectangle $b \in\left(-2^{\circ}, 2^{\circ}\right), \ell \in\left(-10^{\circ}, 0^{\circ}\right)$ (middle panel in Fig. 11). The spectrum of CR electrons is modeled as a power-law function with an exponential cutoff at $1 \mathrm{PeV}$. We separate the SL and IR contributions to the ISRF by formally splitting the ISRF at $0.1 \mathrm{eV}$.

Table C.1. Spectra of CR electrons near the GC relative to the reference model based on the ISRF calculation of Porter et al. (2008) used in GALPROP v54.1.

\begin{tabular}{lcc}
\hline \hline ISRF model & Relative normalization at 1 GeV & Index \\
\hline Porter et al. (2008) & 1 & 2.71 \\
Porter et al. (2017) R12 & 0.59 & 2.61 \\
Porter et al. (2017) F98 & 1.16 & 2.71 \\
Popescu et al. (2017) & 1.64 & 2.79 \\
\hline
\end{tabular}

